\title{
Can We See the Energy Densities? I. Insights from Real-Time Time-Dependent Density Functional Theory Simulations ${ }^{\dagger}$
}

\author{
Junjie Yang, ${ }^{a, \#}$ Zheng $\mathrm{Pe}^{b}{ }^{b, \#}$, Jingheng Deng ${ }^{a}$, Yuezhi Mao ${ }^{c}$, Qin $\mathrm{Wu}^{d}$, Zhibo Yang ${ }^{a}$, \\ Bin Wang ${ }^{e}$, Christine M. Aikens ${ }^{f}$, Wanzhen Liang ${ }^{* b}$, and Yihan Shao*a
}

\begin{abstract}
In this work, we report a scheme to evaluate and analysis of energy density fluctuations during the real-time time-dependent density functional theory (RT-TDDFT) simulations. Using $\mathrm{Ag}_{4}-\mathrm{N}_{2}$ complexes as examples, it is shown that the energy of each molecular fragment (such as $\mathrm{Ag}_{4}$ and $\mathrm{N}_{2}$ ) can be readily computed by partitioning Kohn-Sham energy densities using Becke or fragment-based Hirshfeld (FBH) scheme. A strong orientation-dependence is observed for the energy flow between the $\mathrm{Ag}_{4}$ cluster and a nearby $\mathrm{N}_{2}$ molecule in the RT-TDDFT simulations. Future applications of such an energy density analysis in electron dynamics simulations are discussed.
\end{abstract}

\section{Introduction}

Real-time time-dependent density functional theory (RTTDDFT), 114 which is also known as time-dependent Kohn-Sham (TDKS) theory, has emerged in recent years as a powerful tool in the modeling of various molecular spectra and electron/exciton transfer. $\frac{56}{6}$ For single molecules, RT-TDDFT has been employed to compute the UV/Vis absorption spectra, 4.7 near-edge x-ray absorption spectra,, 10 electronic responses of molecules in strong fields, 211 14 plasmonic excitations of silver and gold nanowires ${ }^{15116}$ and silver nanoclusters, ${ }^{\sqrt{17}}$ molecular conductivity, ${ }^{[18]}$ singlet-triplet gaps, ${ }^{19}$ electron localization functions, ${ }^{2021}$ dynamical hyperpolarizability, ${ }^{22}$ frequency-dependent

\footnotetext{
${ }^{a}$ Department of Chemistry and Biochemistry, University of Oklahoma, 101 Stephenson Pkwy, Norman, OK 73019, United States. E-mail: yihan.shao@ou.edu

${ }^{b}$ State Key Laboratory of Physical Chemistry of Solid Surfaces, Collaborative Innovation Center of Chemistry for Energy Materials, Fujian Provincial Key Laboratory of Theoretical and Computational Chemistry, and Department of Chemistry, College of Chemistry and Chemical Engineering, Xiamen University, Xiamen 361005, P. R. China. E-mail: liangwz@xmu.edu.cn

${ }^{c}$ Department of Chemistry, Stanford University, Stanford, CA 94305, United States.

${ }^{d}$ Center for Functional Nanomaterials, Brookhaven National Laboratory, Upton, NY 11973, United States.

e Center for Interfacial Reaction Engineering and School of Chemical, Biological, and Materials Engineering, Gallogly College of Engineering, University of Oklahoma, Norman, OK 73019, United States.

${ }^{f}$ Department of Chemistry, Kansas State University, Manhattan, KS 66506, United States.

\# These two authors contributed equally to this work.

$\dagger$ Electronic Supplementary Information (ESI) available: [details of any supplementary information available should be included here]. See DOI: 00.0000/00000000.
}

nonlinear optical responses, 23 and electronic circular dichroism. 24 RT-TDDFT has also been widely used to study electron/energy transfer in molecular complexes, such as hydrogen-atom-adsorbed lithium atom clusters, ${ }^{255}$ dye-sensitized titanium oxide clusters, 26 benzaldehyde clusters, 27 ion-irradiated peptide and DNA, 2829 silver nanowire arrays, $\underline{30}$ and nitrogen-molecule-adsorbed silver nanowire. 31

RT-TDDFT simulations capture the time-evolution of KohnSham orbitals and charge density of the system of interest, such as dye molecules,, 7 molecular conductors,,$\frac{118}{118}$ and plasmonic nanoparticles. ${ }^{15}$.1730131 This would allow scientists to "watch" the charge flow between molecule fragments with time. In plasmonmodulated catalysis, $\underline{321}$ for example, "hot" electrons are expected to flow from the plasmonic nanoparticle into the anti-bonding

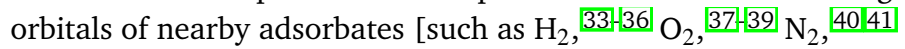
$\mathrm{NH}_{3},{ }^{, 42}$ acetylene, $\frac{\sqrt[36]{6}}{\text { nitrophenol, aminophenols, }, 43}$ and phenylacetylene ${ }^{[44}$ ], thereby chemically activating these molecules.

In this article, we address a simple question: Can we analyze and visualize the energy flow in a RT-TDDFT simulation that accompanies the electron charge flow? To the best of our knowledge, no protocol currently exists for visualizing such energy flow. Fortunately, as shown in this article, such a task is rather straightforward given the Kohn-Sham density functional theory (KS-DFT) energy distribution, which was originally proposed by Nakai et al to obtain atomic and bond energy components. ${ }^{45}$.47 Thereby, via computing the Kohn-Sham energy density distribution along a RT-TDDFT trajectory, it becomes feasible to monitor the energy flow within a single molecule or between different fragments of a molecular complex. 
This manuscript is organized as follows. In Section 2, a brief summary of the RT-TDDFT methodology will be provided, followed by a re-introduction of Kohn-Sham energy density as proposed originally by Nakai et al.45 47 Section 2.2 also includes a formula for expressing the TDKS state as a superposition of the KohnSham ground-state and linear-response time-dependent density functional theory (LR-TDDFT) 52 excited states, with a detailed discussion in the Appendix. Implementational and computational details are described in Section 3. In Section 4, we model the RTTDDFT energy density of $\mathrm{Ag}_{4}-\mathrm{N}_{2}$ complexes. To help understand the real-time fluctuation of the Kohn-Sham charge and energy densities, a representation of the time-evolving Kohn-Sham determinant "wavefunction" as a superposition of adiabatic electronic states will be employed. A discussion will be provided in Section 5 , with an emphasis on how to perturb a molecular system to generate substantial charge and energy flow between molecular fragments. Concluding remarks will be made in Section 6. In an accompanying paper, ${ }^{53}$ we report the energy densities in LR-TDDFT calculations.

\section{Methodology}

\subsection{One-Electron Basis}

Throughout this article, we adopt the orthonormal one-electron basis spanned by the canonical Kohn-Sham molecular orbitals (CMO) of the unperturbed system, which are all real and denoted by $\chi_{p}$, $\chi_{q}, \chi_{r}$, and $\chi_{s}$. Furthermore, $\chi_{i}, \chi_{j}, \chi_{k}$, and $\chi_{l}$, represent occupied CMOs, while $\chi_{a}, \chi_{b}, \chi_{c}$, and $\chi_{d}$ stand for virtual (i.e. unoccupied) CMOs. One-electron core-Hamiltonian integrals between these CMOs are

$$
h_{p q}=\int \chi_{p}(\mathbf{r}) \hat{h} \chi_{q}(\mathbf{r}) d \mathbf{r}
$$

where $\hat{h}$ contains both kinetic energy and nuclear attractions. The dipole moment matrices are

$$
\vec{\mu}_{p q}=\left[\int \chi_{p}(\mathbf{r}) x \chi_{q}(\mathbf{r}) d \mathbf{r}, \int \chi_{p}(\mathbf{r}) y \chi_{q}(\mathbf{r}) d \mathbf{r}, \int \chi_{p}(\mathbf{r}) z \chi_{q}(\mathbf{r}) d \mathbf{r}\right]
$$

Two-electron repulsion integrals are

$$
(p q \mid r s)=\iint \chi_{p}(\mathbf{r}) \chi_{q}(\mathbf{r}) \frac{1}{\left|\mathbf{r}-\mathbf{r}^{\prime}\right|} \chi_{r}\left(\mathbf{r}^{\prime}\right) \chi_{s}\left(\mathbf{r}^{\prime}\right) d \mathbf{r} d \mathbf{r}^{\prime}
$$

The electrostatic potential of each pair of CMOs is

$$
V_{p q}(\mathbf{r})=\int \frac{\chi_{p}\left(\mathbf{r}^{\prime}\right) \chi_{q}\left(\mathbf{r}^{\prime}\right)}{\left|\mathbf{r}-\mathbf{r}^{\prime}\right|} d \mathbf{r}^{\prime}
$$

\subsection{Many-Electron Basis for Superposition Analysis}

In the weak-field limit, a RT-TDDFT electronic state can schematically be written as a first-order perturbation to $\Phi_{0}$, the KS-DFT electronic ground state of an unperturbed system,

$$
\Phi(t)=\Phi_{0}+\sum_{m} a_{m}(t) \Psi^{(m)}
$$

Here $\Psi^{(m)}$ are open-shell singlet excited states of the same system within linear response time-dependent density functional theory (LR-TDDFT). ${ }^{48}$ Namely, they correspond to solutions to a gen- eralized eigenproblem,

$$
\left(\begin{array}{ll}
\mathbf{A} & \mathbf{B} \\
\mathbf{B} & \mathbf{A}
\end{array}\right)\left(\begin{array}{l}
\mathbf{X} \\
\mathbf{Y}
\end{array}\right)=\omega\left(\begin{array}{c}
\mathbf{X} \\
-\mathbf{Y}
\end{array}\right)
$$

where matrices $\mathbf{A}$ and $\mathbf{B}$ couple singly-excited electronic configurations $\left(\Phi_{i}^{a}\right), 480 \mathbf{X}^{(m)}$ and $\mathbf{Y}^{(m)}$ (i.e. $m$-th column of $\mathbf{X}$ and $\mathbf{Y}$ matrices) represent the amplitudes for the $m$-th excited state, and $\omega^{(m)}$ is the corresponding excitation energy. For each state, the transition dipole moment is

$$
\vec{\mu}^{(0, m)}=\sum_{a i}\left(X_{a i}^{(m)}+Y_{a i}^{(m)}\right) \vec{\mu}_{a i}
$$

The orthonormality between these singlet excited states is

$$
(\mathbf{X}+\mathbf{Y})^{(m)} \cdot(\mathbf{X}-\mathbf{Y})^{(n)}=\sum_{a i}\left(X_{a i}^{(m)}+Y_{a i}^{(m)}\right)\left(X_{a i}^{(n)}-Y_{a i}^{(n)}\right)=\delta_{m n}
$$

Mathematically, we can write the identity matrix in the subspace of LR-TDDFT open-shell singlet excited states of the unperturbed system as 54

$$
\left(\mathbf{I}^{\mathrm{LR}-\mathrm{TDDFT}}\right)_{a i, b j}=\sum_{m}\left(X_{a i}^{(m)}+Y_{a i}^{(m)}\right)\left(X_{b j}^{(m)}-Y_{b j}^{(m)}\right)
$$

which can be used (see Eqs. A14 and A15) to derive the superposition coefficients $\left(a_{m}\right)$ in Eq. 5

Accordingly, the KS-DFT response kernel is 54

$$
\left[(\mathbf{A}+\mathbf{B})^{-1}\right]_{a i, b j}=\sum_{m} \frac{1}{\omega^{(m)}}\left(X_{a i}^{(m)}+Y_{a i}^{(m)}\right)\left(X_{b j}^{(m)}+Y_{b j}^{(m)}\right)
$$

\subsection{RT-TDDFT Energy and Propagation}

Within RT-TDDFT, the electronic state at a given time $t$ is described by the occupied Kohn-Sham orbitals

$$
\psi_{i}(\mathbf{r}, t)=\sum_{p} C_{p i} \chi_{p}(\mathbf{r})
$$

and the corresponding electron density,

$$
\rho(\mathbf{r}, t)=\sum_{p q} P^{p q}(t) \chi_{p}(\mathbf{r}) \chi_{q}(\mathbf{r})
$$

where the one-particle density matrix

$$
P^{p q}=\sum_{i} C_{p i} C_{q i}^{*}
$$

contain both real and imaginary blocks.

The KS-DFT energy at a given time $t$ contains several components: nuclear-nuclear repulsion ( $\left.E_{\text {nuc-rep }}\right)$, core-electron ( $h$, a sum of kinetic $(T)$ and nuclear attraction $(N)$ energies), Coulomb $(J)$, Hartree-Fock exchange $(K)$, and exchange-correction (XC),

$$
\begin{aligned}
E(t)= & E_{\text {nuc-rep }}+\sum_{p q} P^{p q}(t) h_{p q}+\frac{1}{2} \sum_{p q} \sum_{r s} P^{p q}(t)(p q \mid r s) P^{r s}(t) \\
& -\frac{a_{K}}{2} \sum_{p q, r s} P^{p q}(t)(p r \mid q s) P^{r s}(t)+E_{\mathrm{xc}}[\rho(\mathbf{r}, t)]
\end{aligned}
$$


where $a_{K}$ is the ratio of Hartree-Fock exchange for the given functional, and $E_{\mathrm{xc}}[\rho(\mathbf{r}, t)]=\int f_{\mathrm{xc}}[\rho(\mathbf{r}, t)] d \mathbf{r}$ is the exchange-correlation functional. Note that the imaginary part of the density matrix only contributes to the Hartree-Fock exchange energy term. 55

At each time step, one can build the Fock matrix, $\mathscr{F}[\mathbf{P}(t)]$,

$$
\begin{aligned}
F_{p q}(t)= & h_{p q}+\sum_{s t}(p q \mid r s) P^{r s}(t)-a_{K} \sum_{r s}(p r \mid q s) P^{r s}(t) \\
& +\int \chi_{p}(\mathbf{r}) \frac{\delta E_{\mathrm{xc}}}{\delta \rho(\mathbf{r}, t)} \chi_{q}(\mathbf{r}) d \mathbf{r}
\end{aligned}
$$

and use it to drive the time-evolution of the density matrix

$$
i \frac{\partial}{\partial t} \mathbf{P}(t)=[\mathbf{F}(t), \mathbf{P}(t)]
$$

\subsection{Grid-Based RT-TDDFT Energy Density}

The electronic portion of the KS-DFT energy in Eq 14 can be evaluated via a real-space integration,

$$
E(t)=E_{\text {nuc-rep }}+\int \rho^{E}(\mathbf{r}, t) d \mathbf{r}
$$

with the energy density $\rho^{E}(\mathbf{r})$ composed of the following components,

$$
\begin{aligned}
\rho^{E}(\mathbf{r}, t) & =\rho^{T}(\mathbf{r}, t)+\rho^{N}(\mathbf{r}, t)+\rho^{J}(\mathbf{r}, t) \\
& +a_{K} \rho^{K}(\mathbf{r}, t)+\rho^{\mathrm{XC}}(\mathbf{r}, t)
\end{aligned}
$$

Since the nuclear repulsion energy, $E_{\text {nuc-rep }}$, cannot be decomposed on a real-space grid, it is not included in the energy density. Strictly speaking, the energy density in Eq. 18 should be called "electronic energy density".

In Eq 18, the first two components, kinetic and nuclear attraction energy densities, can be written as,

$$
\begin{aligned}
& \rho^{T}(\mathbf{r}, t)=\frac{1}{2} \sum_{p q} P_{p q}(t)\left[\nabla \chi_{p}(\mathbf{r})\right] \cdot\left[\nabla \chi_{q}(\mathbf{r})\right] \\
& \rho^{N}(\mathbf{r}, t)=-\rho(\mathbf{r}, t) \sum_{A} \frac{Z_{A}}{|\mathbf{r}-\mathbf{A}|}
\end{aligned}
$$

The well-known kinetic energy density in Eq. 19 is used in the formulation of, for example, popular TPSS and M06-series functionals. .5657 The Coulomb $(J)$ and Hartree-Fock exchange $(K)$ components can be evaluated as,

$$
\begin{gathered}
\rho^{J}(\mathbf{r}, t)=\sum_{p q, r s} P^{p q}(t) P^{r s}(t) \chi_{p}(\mathbf{r}) \chi_{q}(\mathbf{r}) V_{r s}(\mathbf{r}) \\
\rho^{K}(\mathbf{r}, t)=-\sum_{p q, r s} P^{p q}(t) P^{r s}(t) \chi_{p}(\mathbf{r}) \chi_{r}(\mathbf{r}) V_{q s}(\mathbf{r})
\end{gathered}
$$

using the electrostatic potential of each $\mathrm{CMO}$ pair in Eq4. Note that the Hartree-Fock exchange energy density is used in the formulation of the Becke'05 functional and its variants. $\frac{[58 \cdot 60}{6 a s t l y,}$ the exchange-correlation energy density is just the functional in the numerical integration,

$$
\rho^{\mathrm{XC}}(\mathbf{r}, t)=f_{\mathrm{xc}}(\rho(\mathbf{r}, t))
$$

\section{Computational Details}

The evaluation of grid-based RT-TDDFT energy density, as described above in Section 2.4, was implemented in a development version of the PYSCF software package.61 Also implemented were fragment-based Hirshfeld (FBH) ${ }^{62}$ and Becke population schemes ${ }^{63}$ (see Section 2.3 in the accompanying paper ${ }^{[53]}$ for details) for partitioning the RT-TDDFT charge and energy densities. Energy densities were evaluated on two sets of grid points. A cubic grid was used for visualization purposes, whereas an atomcentered grid ${ }^{63}$ was used for FBH or Becke integrations over the fragments.

Several functionals (such as PBE, ${ }^{64} \mathrm{PBE}, 0{ }^{65} \mathrm{~B} 3 \mathrm{LYP}, 66,68$ and $\omega \mathrm{B} 97 \mathrm{X}-\mathrm{D}{ }^{69}$ ) are supported in our implementation. Only PBE0 results are presented in the next section, while the use of other functionals was found to lead to qualitatively similar results for the test systems. Stuttgart effective core potential and basis set ${ }^{70}$ were employed for silver atoms, and 6-31G(d) basis functions were used on nitrogen atoms. (a)
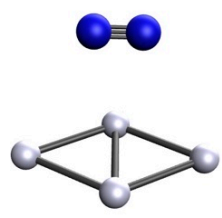

(b)
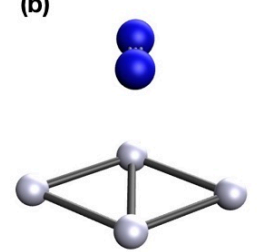

(c)
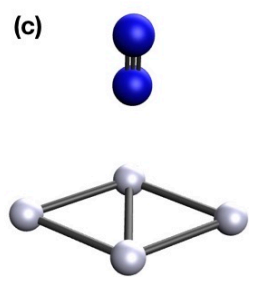

Fig. 1 Three $\mathrm{Ag}_{4}-\mathrm{N}_{2}$ complexes studied in this work: a) stacked-long; b) stacked-short; and c) T-shape.

In this study, three different configurations of $\mathrm{Ag}_{4}-\mathrm{N}_{2}$ complexes were used as model systems for interfacial energy and charge transfer. Previously, $\mathrm{Ag}_{4}$ and other small silver clusters and their interactions with guanine molecule were investigated by Dale, Senanayake, and Aikens. ${ }^{71}$ As shown in Fig. 11, in the first two of our configurations, the nitrogen molecules were stacked on top of the $\mathrm{Ag}_{4}$ cluster, with the $\mathrm{N} \equiv \mathrm{N}$ bond lying in parallel with the long axis of $\mathrm{Ag}_{4}$ (labelled as "stacked-long") or the short axis (labelled as "stacked-short"). In the third configuration, the nitrogen molecule formed a T-shaped complex with $\mathrm{Ag}_{4}$ (labelled as "T-shape").

Out of the many RT-TDDFT propagation schemes 1 1/455/72, 76 developed over the years, we have implemented the modified midpoint unitary transform (MMUT) scheme, ${ }^{2}$ exponential density predictor/corrector (EP-PC) and linear Fock linear density predictor/corrector (LFLP-PC). ${ }^{76}$ In addition, we proposed a series of approximate mid-point unitary transform (AMUT) algorithms. These schemes are illustrated in Fig. S1 of the ESI. For EP-PC and LFLP-PC, a convergence threshold of $\xi=10^{-7}$ au was adopted. Several time steps were tested for the orbital/density propagation, with results shown only with two time steps ( 0.05 and $0.50 \mathrm{au})$ in the next section. All our RT-TDDFT calculations were initialized with a fully-converged SCF solution under a weak-field perturbation (field strength is $10^{-3} \mathrm{au}$, in the $x$-direction which points from $\mathrm{Ag}_{4}$ to $\mathrm{N}_{2}$ fragment). Then the density matrices were propagated without an external field within the basis of canonical MOs of the 
unperturbed system. For all three complexes, the total propagation time is 200.0 au (i.e. $4.83 \mathrm{fs}$ ).

For the superposition analysis of the RT-TDDFT charge and energy densities, we started from the initial density matrix, $\mathbf{P}(0)$, for a weakly-perturbed $\mathrm{Ag}_{4}-\mathrm{N}_{2}$ complex (as represented in the basis of canonical MOs of the unperturbed system). Its inner product with the amplitudes for the $m$-th open-shell singlet state (Eq. A15) yielded its superposition coefficient, $\tilde{a}_{m}(0)$. From the coefficients for the first 200 open-shell singlet states, we then approximated the time-dependent orbital rotations, $\boldsymbol{\Theta}(t)$, using Eq. A20 and the corresponding density matrix, $\mathbf{P}(t)$, using Eq. A7. The electron and energy densities were then generated from $\mathbf{P}(t)$ following Eqs. 12 and 18 and subjected to FBH and Becke decomposition analyses.

\section{Results}

\subsection{Ground State Energy Density of $\mathrm{N}_{2}$ and $\mathrm{Ag}_{4}$}
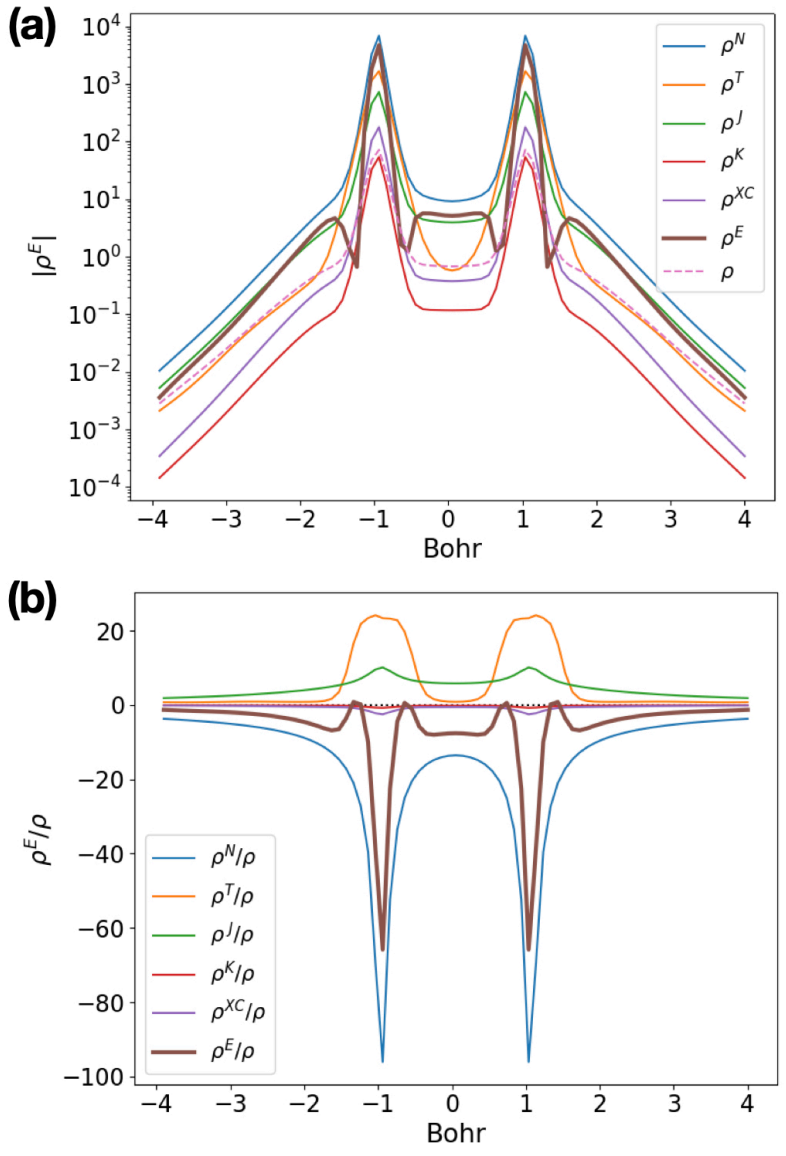

Fig. 2 Ground-state electron and energy densities of $\mathrm{N}_{2}$ molecule along the $\mathrm{N}-\mathrm{N}$ axis. (a) The absolute value of all energy components and electron density; and (b) the ratio of different energy components to the electron density. Obtained from KS-DFT calculations using the PBE0 functional and 6-31G(d) basis set.

Before proceeding to analyze the RT-TDDFT energy densities, let us use $\mathrm{N}_{2}$ as a model compound to gain some basic understandings about the ground-state energy density of molecular systems. First, one would expect all energy density components to have singular values at the nuclear positions and decay exponentially away from the nuclei, just like the electron density. This was confirmed in Fig. 2a, which showed (the absolute value of) all energy density components along the molecular axis of $\mathrm{N}_{2}$.

Second, one might expect these energy components to exhibit slightly different decay rates in comparison to the electron density. While one can check this by examining the decay behavior in Fig. 2 a, a better way is to plot the ratio between a given energy density component and the electron density along the molecular axis. Indeed, as clearly shown in Fig. 2p, the nuclear attraction energy density $\left(\rho^{N}\right)$, kinetic energy density $\left(\rho^{T}\right)$, and the Coulomb energy density $\left(\rho^{J}\right)$ all decayed noticeably faster than the electron density. In Fig. 2. Hartree-Fock exact exchange energy density $\left(\rho^{K}\right)$ and the exchange-correlation energy density $\left(\rho^{X C}\right)$ also decayed faster than the electron density, but they were substantially smaller than other three energy components.
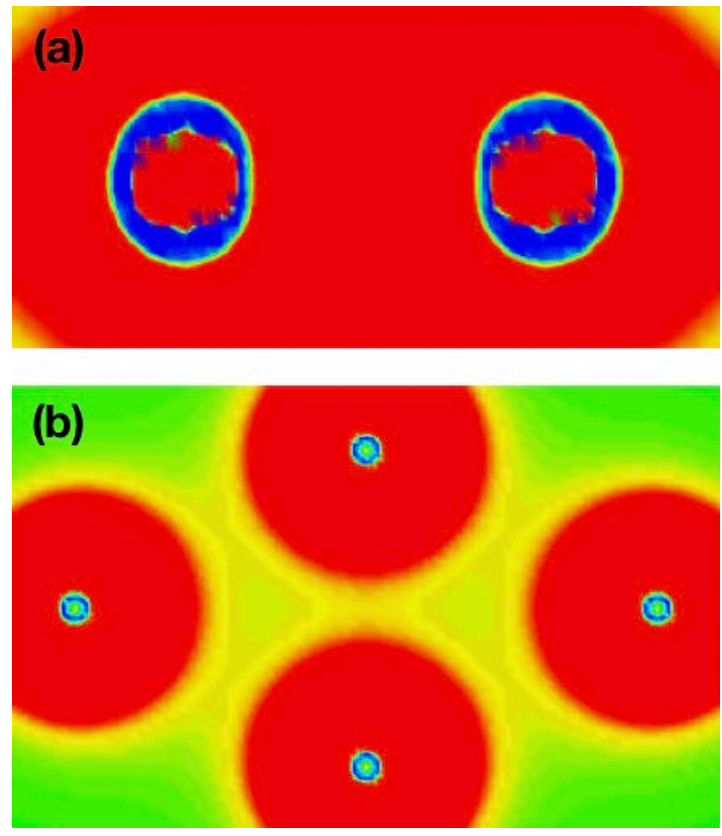

Fig. 3 Ground-state energy density (blue = positive; red = negative) of a) $\mathrm{N}_{2}$ and b) $\mathrm{Ag}_{4}$ molecules. Obtained from KS-DFT calculations using the PBE0 functional and 6-31G(d) basis set.

Third, we would expect the nuclear attraction energy density $\left(\rho^{N}\right)$ to stay negative throughout the real space, while the kinetic energy density $\left(\rho^{T}\right)$ and the Coulomb energy density $\left(\rho^{J}\right)$ remain positive. This was fully confirmed in Fig. 2 2 . In fact, these three energy density components contributed the most to the total energy density. Furthermore, a dominating negative $\rho^{N}$ value led to a net negative value for total energy density at most positions along the molecular axis. A 2-dimensional plot in Fig. 3 B also clearly showed a negative value (colored in red) for the $\mathrm{N}_{2}$ total energy density at most grid points along or away from the molecular axis.

However, Fig. $2 \mathrm{~b}$ also indicated that, in the region close to the nucleus, $\rho^{N}$ decayed faster than $\rho^{T}$ and $\rho^{J}$ combined. This produced a thin positive-valued shell around each nitrogen atom, which appeared as thin "blue donuts" in Fig. 33. While mathe- 

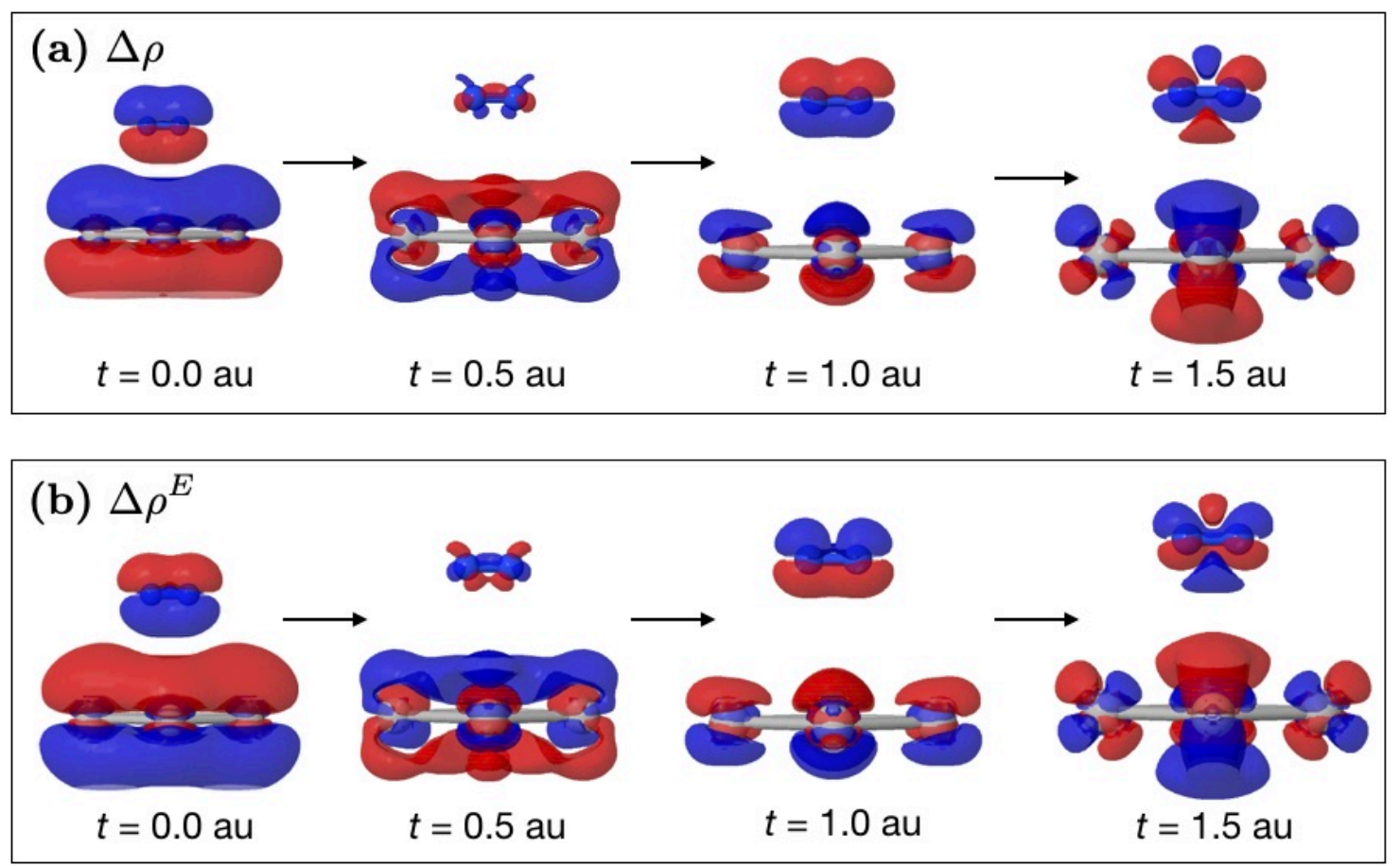

Fig. 4 Time evolution of (a) the electron density; and (b) the energy density (blue = positive) of the $\mathrm{Ag}_{4}-\mathrm{N}_{2}$ complex in the "stacked-long" configuration. Only the differences against the ground-state densities were shown. Isovalues were $2.0 \times 10^{-5}$ au for electron densities, and $2.0 \times 10^{-4}$ au for energy densities. Obtained from RT-TDDFT calculations using the LFLP-PC propagator, PBE0 functional, and 6-31G(d) basis set.

matically intriguing, these positive energy density regions do not seem to have much physical significance, at least in the context of our analysis of the RT-TDDFT energy density fluctuation. Further, we suspect that such positive energy density regions might be an artifact from using Gaussian basis functions, whose low curvature can lead to wrong asymptotic behavior near the nuclei for the kinetic energy density .

The ground-state energy density for $\mathrm{Ag}_{4}$, another component of our $\mathrm{Ag}_{4}-\mathrm{N}_{2}$ complexes, was shown in Fig. 3 b. Similar to $\mathrm{N}_{2}$, the ground-state energy density of $\mathrm{Ag}_{4}$ was found to be negative in most regions, which was consistent with an overall negative electronic energy. On the other hand, a positively-valued shell also appeared around each silver atom, albeit thinner and much closer to the nuclei.

\subsection{Choice of RT-TDDFT density matrix propagators}

The several different RT-TDDFT propagation schemes tested in this work, such as MMUT, EP-LP, LFLP-PC, and approximate mid-point unitary transform (AMUT), are similar in that they are all exact to the second order, meaning that the leading error is $\mathscr{O}\left((\Delta t)^{3}\right)$. For the $\mathrm{Ag}_{4}-\mathrm{N}_{2}$ complexes, however, these schemes exhibit rather different behaviors for the conservation of the total energy.

The MMUT scheme from Li et al was developed as the first second-order algorithm for RT-TDDFT simulations. ${ }^{[2}$ Indeed, when a 0.05 au time step is used, MMUT well conserved the energy of the complex to within $1.0 \times 10^{-8}$ au during the 200 au of the simulation (see Figure S2a). While a systematic drift in the MMUT energies could be observed over the simulation period, it was largely caused by our simple implementation of the scheme. In more sophisticated implementations of MMUT, 77 the trajectory can be reset periodically, thus avoiding such a systematic energy drift.

Using a 0.05 au time step, AMUT-3, another approximate unitary transformation scheme but requiring four Fock builds during each time step (illustrated in Figure S1b), also displayed an energy drift, though at a smaller scale (see Figure S2a). Among the two predictor/corrector schemes from Zhu and Herbert, ${ }^{[76}$ EP-PC produced a large fluctuation, even with a 0.05 au timestep. LFLPPC, on the other hand, conserved the energy well with a net shift of $7.0 \times 10^{-12}$ au during the simulation.

When the time step is increased to $0.5 \mathrm{au}$, the four propagation schemes followed a similar trend in terms of their performance. As shown in Figure S2b, our implementation of MMUT and AMUT-3 led to a substantial energy change during the 200 au simulation period. EP-PC energies fluctuated up to $1.5 \times 10^{-7}$ au around the initial energy, while LFLP-PC conserved the energy well with a similar net shift of $7.0 \times 10^{-12}$ au during the simulation.

Based on the performance of four propagation schemes discussed here, the LFLP-PC scheme was adopted to obtain results in the remainder of this article. On average, it required 3 and 8 predictor-corrector steps with 0.05 and 0.50 au timesteps, respectively.

\subsection{Fluctuation of Different RT-TDDFT Energy Components}

In general, the RT-TDDFT energy contains five components in $\mathrm{Eq} 18$. In a calculation with effective core potentials (ECP) on the metal atoms, an additional ECP component will be involved. In contrast to the total energy, which should be conserved along 

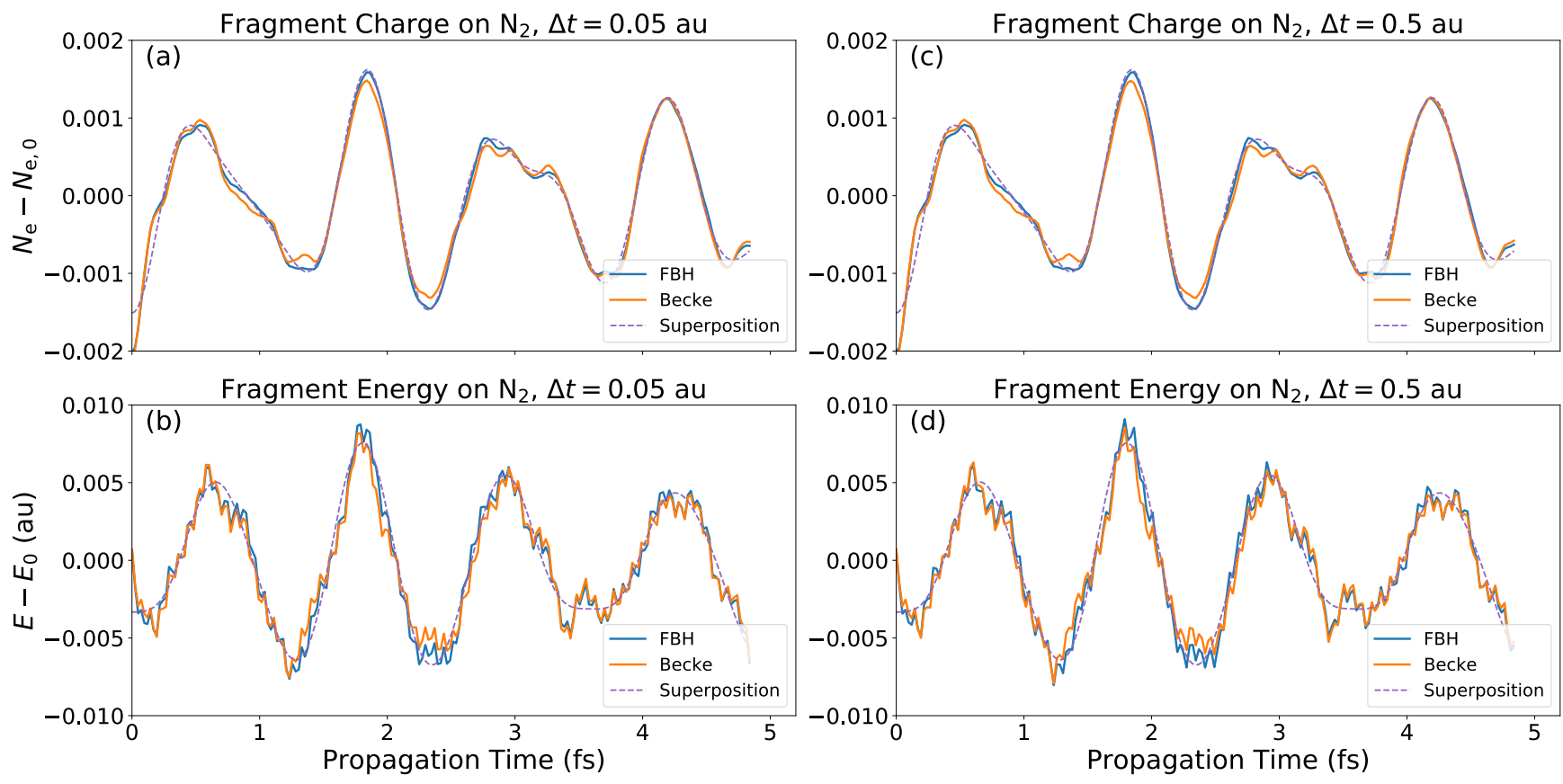

Fig. 5 Time evolution of $(a, b)$ the fragment charge and $(c, d)$ fragment energy of $\mathrm{N}_{2}$ molecule in the "stacked-long" configuration of the $\mathrm{Ag}_{4}-\mathrm{N}_{2}$ complex. Obtained from RT-TDDFT simulation using the PBE0 functional.

a RT-TDDFT trajectory, individual energy components are shown in Figures S3, S4, and S5 to fluctuate with time for three $\mathrm{Ag}_{4}-$ $\mathrm{N}_{2}$ complexes. In particular, the nuclear attraction energy $\left(E_{V}\right)$ and Coulomb energy $\left(E_{J}\right)$ oscillate the most (up to $1.0 \times 10^{-2} \mathrm{au}$ ) along the trajectory. The kinetic energy density $\left(E_{T}\right)$ also has a substantial fluctuation up to $1.0 \times 10^{-3}$ au. All other three energy components, the Hartree-Fock exchange energy $\left(E_{K}\right)$, the exchange-correlation energy $\left(E_{\mathrm{XC}}\right)$, and the ECP energy $\left(E_{\mathrm{ECP}}\right)$, has a smaller fluctuations up to $1.0 \times 10^{-4} \mathrm{au}$.

Due to the unique format of ECP projection operators, 707879 the ECP energy cannot be decomposed onto a real-space grid in a straightforward way. But, given the small fluctuation of this energy component, the corresponding ECP energy density was omitted in the analysis below.

\subsection{Charge/Energy Transfer Along RT-TDDFT Trajectories}

In our simulations of each $\mathrm{Ag}_{4}-\mathrm{N}_{2}$ complex, a weak-field perturbation (field strength: $10^{-3}$ au) was applied in the direction from $\mathrm{Ag}_{4}$ to $\mathrm{N}_{2}$ fragment at time zero. After a fully-converged SCF solution was reached, the Kohn-Sham orbitals and density matrix were propagated in a field-free environment. Since the initial state was prepared using a weak field, the RT-TDDFT "wavefunction" can be interpreted as a superposition of the Kohn-Sham electronic ground-state and LR-TDDFT open-shell singlet excited states as shown in Eq. 5. where the expansion coefficient can be obtained using Eq. A15 or A12.

Various energy density components along the RT-TDDFT trajectories were evaluated on the atom-centered grid according to Eqs. 19, 23. They were added together to yield the total energy densities, which were subsequently partitioned into the two fragments $\left(\mathrm{Ag}_{4}\right.$ and $\left.\mathrm{N}_{2}\right)$ using fragment-based Hirshfeld $(\mathrm{FBH})$ and
Becke schemes (see Section 2.3 in the accompanying paper ${ }^{53}$ ).

For the stacked-long configuration of $\mathrm{Ag}_{4}-\mathrm{N}_{2}$, the time-evolution of the total charge and energy densities (with reference to the ground-state values) within the first 1.5 au were shown in Figure 4 Clearly, the two densities evolved in sync with each other, with the energy density fluctuation always having an opposite sign as the charge density. This was expected because, when a region gained more electrons (colored blue in Figure $4 \mathrm{a}$ ), the energy density became "higher" (i.e. more negative, shown in red in Figure 4p).

Through FBH and Becke partitioning, fragment charge and energy on $\mathrm{N}_{2}$ molecule were obtained and their fluctuations are shown in Figure 5 The actual fluctuation patterns clearly did not depend on either the partitioning scheme (FBH or Becke) or the time step ( $0.05 \mathrm{au}$ or $0.5 \mathrm{au}$ ) in use, attesting the robustness of the LFLP-PC propagator. Observing this, we only showed results with a 0.5 au time step for two other complexes.

In Figure 5, the fragment charge/energy densities clearly fluctuated in sync with each other. (Note that panels a and c showed the net number of electrons on the $\mathrm{N}_{2}$ molecule, which is consistent with Figure 4h.) Essentially, when more electrons were located on the $\mathrm{N}_{2}$ molecule, they occupied the unoccupied orbitals of the molecule, thus increasing its fragment energy.

Also in Figure 5, the fluctuation pattern as predicted using the superposition picture (including up to 200 open-shell singlet excited states in the superposition) was shown to well reproduce FBH and Becke ones (based on actual RT-TDDFT density matrices), thus validating our superposition analysis. In general, as indicated by Eq. A12 only excited states with a substantial transition dipole moment along the direction of the initial electric field can participate in the superposition. For our current test case, only those states (with $p_{m}>0.01$ ) were listed in Table S1. The oscillation 

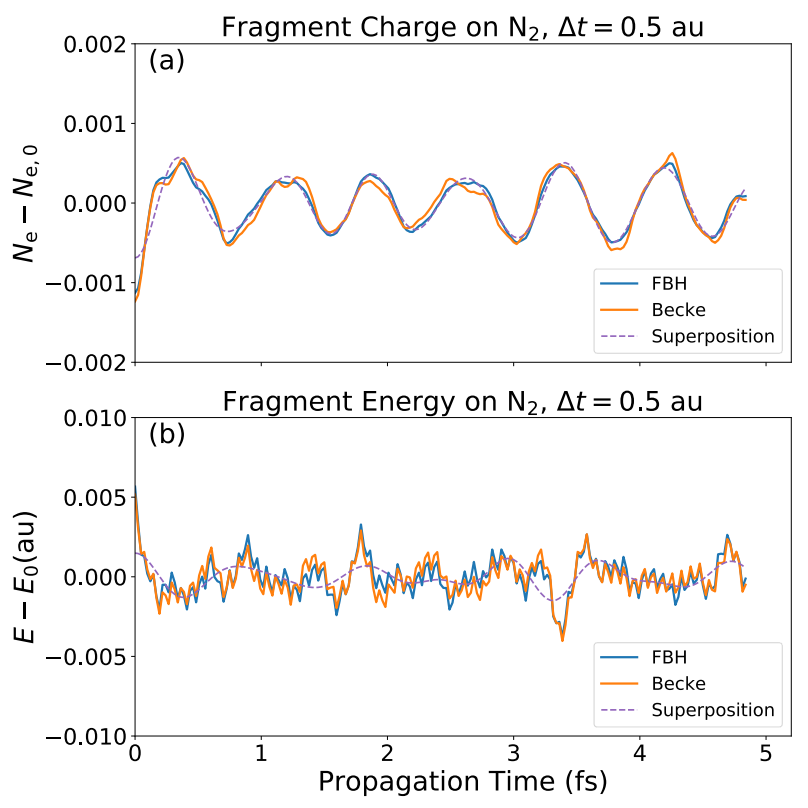

Fig. 6 Time evolution of (a) the fragment charge and (b) fragment energy of $\mathrm{N}_{2}$ molecule in the "stacked-short" configuration of the $\mathrm{Ag}_{4}-\mathrm{N}_{2}$ complex. Obtained from RT-TDDFT simulation using the PBE0 functional.

period of around $1.2 \mathrm{fs}$ for the fragment charge/energy densities then can be traced to the 5-th open-shell singlet excited state of the $\mathrm{Ag}_{4}-\mathrm{N}_{2}$ complex. As shown in Table S1, this excited state has an excitation energy of $0.1263 \mathrm{au}$, corresponding to a period of $1.2032 \mathrm{fs}$. Several higher excited states also contributed to the superposition "wavefunction" at each time step, leading to the fine features of the charge/energy fluctuations in Figure 5

Surprisingly, it was the T-shape configuration (rather than the stacked-short one) that more closely resembled the stacked-long configuration. For instance, the fragment charge fluctuation for the T-shape complex in Figure $7 \mathrm{~b}$ appeared very similar to those in Figure 5 . This happened because, as shown in Tables S1 and S3, excited states with similar excitation energies from the two configurations became "occupied" (i.e. had significant expansion coefficients) at time zero upon the perturbation by the weak field. On the other hand, the stacked-short configuration was shown in Figure 6 to exhibit a faster oscillation of the fragment charge, largely because the leading excited-state contributions (12-th and 34-th) had periods of 0.9487 and $0.7627 \mathrm{fs}$.

\section{Discussion}

In the catalysis of an adsorbate reaction on a metal nanoparticle, the chemical bonds in the adsorbate (such as the $\mathrm{N} \equiv \mathrm{N}$ bond in the $\mathrm{N}_{2}$ molecule) are weakened by injecting electrons to partially occupy its antibonding orbitals. A key challenge is then how to selectively (and partially) populate some excited state(s) to achieve a substantial electron flow to the adsorbate molecule.

In our RT-TDDFT simulations, a fluctuation in fragment charges was indeed observed for the model $\mathrm{Ag}_{4}-\mathrm{N}_{2}$ complexes. To understand such fluctuations at the weak-field limit, a TDKS state was expressed as a superposition of the electronic ground state and open-shell singlet excited states. Specifically, the time-evolving
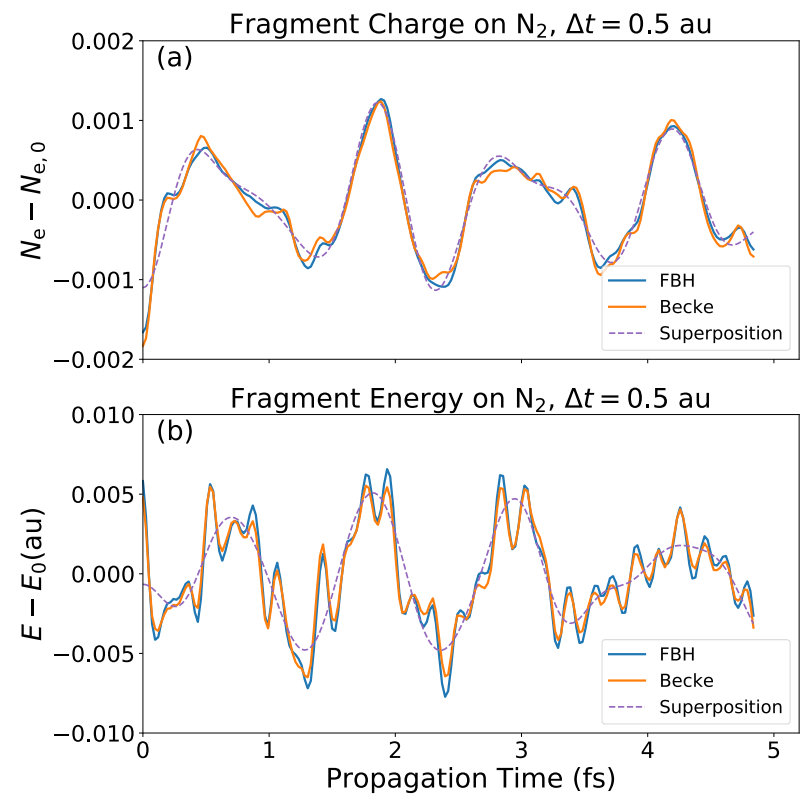

Fig. 7 Time evolution of (a) the fragment charge and (b) fragment energy of $\mathrm{N}_{2}$ molecule in the "T-shape" configuration of the $\mathrm{Ag}_{4}-\mathrm{N}_{2}$ complex. Obtained from RT-TDDFT simulation using the PBE0 functional.

TDKS density matrix $\mathbf{P}(t)$ in Eq. A7 could be readily obtained from the instantaneous orbital rotations $\boldsymbol{\Theta}(t)$ in Eq. A20, where the real component is a linear combination of excited-state transition density matrices $(\mathbf{X}+\mathbf{Y})$ in CMO representation. In other words, the leading excited-state contribution to the TDKS electron density came from the excited state transition densities, each of which weighted by coefficient $a_{m}$. Therefore, when it comes to selecting which excited states to (partially) occupy, one should focus on the fragment decomposition of excited-state transition densities instead of difference densities (i.e. detachment/attachment densities, hole-particle densities).

In contrast to such a simple prediction of the fragment charge fluctuation from the excited state transition densities, though, there is no easy way yet to predict the observed fragment energy fluctuation from the initial superposition. Such energy fluctuation arises from an interplay among all energy components in Eq 18 which are not directly connected to the excitation energy density components described in the accompanying article. ${ }^{53}$

\section{Conclusions}

In this work, starting from Nakai et al's concept of grid-based KohnSham energy density, we evaluated and visualized the fluctuation of the total energy density (and its individual components) along an RT-TDDFT trajectory. This complements the widely-studied motion of electron densities in RT-TDDFT simulations.

After a weak-field perturbation to three $\mathrm{Ag}_{4}-\mathrm{N}_{2}$ model complexes, their fragment energies on the adsorbate $\left(\mathrm{N}_{2}\right)$ were found to oscillate in sync with the charge density. This was explained by expressing the time-evolving TDKS density matrix as a superposition of the ground-state KS-DFT state and LR-TDDFT open-shell singlet states. Based on this interpretation, it was suggested that we can focus on selected open-shell singlet states, whose transition 
density has a substantial partitioning on the adsorbate, in order to steer an effective transfer of the electronic charge towards the adsorbate to weaken its chemical bonds.

On the other hand, our work has several limitations that we shall address in the future. First and foremost, we have focused on RTTDDFT simulations, where the molecular complex (consisting of a metal nanocluster and an adsorbate) adopts a fixed geometry. This analysis needs to be extended to mixed electron/nuclear dynamics simulations, ${ }^{8182}$ such as Ehrenfest dynamics, ${ }^{83}$.85 where nuclear motions enable chemical reactions to occur on the adsorbate.

Secondly, the computational cost of evaluating the TDKS energy density, especially the exact Hartree-Fock energy component, 58,60 will become prohibitively high beyond the small model systems. This arises because various energy density components were computed using exact integrals and a fine numerical integration grid. In order to make our energy density analysis feasible to larger systems, the resolution-of-the-identity (RI) approximation 596086 and other techniques are needed to speed up the computation.

Lastly, the superposition-state analysis was limited to a simple RT-TDDFT simulation, where the system was prepared by applying a weak static electric field at time zero. Further study is needed to better understand the superposition of electronic states following a strong perturbation or a frequency-dependent electrostatic perturbation.

\section{Conflicts of interest}

There are no conflicts to declare.

\section{Acknowledgements}

YS thanks Drs. John Herbert, Aurelian de la Lande, Xiaosong Li, and Weitao Yang for helpful discussions. YS is supported by the National Institutes of Health (grant: R01GM135392), Oklahoma Center for the Advancement of Science and Technology (grant: HR18-130), and the Office of the Vice President of Research and the College of Art and Sciences at the University of Oklahoma (OU). WL acknowledges financial support from the National Natural Science Foundation of China (Grant Nos. 21573177 and 21833006). BW acknowledges funding from the US Department of Energy (Grant No. DE-SC0020300). CMA is supported by the Department of Energy under grant DE-SC0012273. The computing for this project was performed at the OU Supercomputing Center for Education \& Research (OSCER) and the Center of Functional Nanomaterials (CFN). CFN is a U.S. DOE Office of Science Facility, at the Brookhaven National Laboratory under Contract No. DE-SC0012704.

\section{APPENDIX. RT-TDDFT with a Weak Static Electric Field at $t=0$}

\section{A1. Orbital Rotations in a RT-TDDFT Simulation}

As mentioned in Section 2.1. we use the KS-DFT canonical MOs for an unperturbed system as the one-electron basis. In such a basis, the RT-TDDFT orbitals of the unperturbed system would evolve as

$$
\mathbf{C}^{\text {(up) }}(t)=\exp \left(\begin{array}{cc}
-i \mathbf{F}_{\mathrm{oO}}^{\text {(up) }} t & 0 \\
0 & -i \mathbf{F}_{\mathrm{vv}}^{\text {(up) }} t
\end{array}\right)
$$

where $\mathbf{F}_{\mathrm{oO}}^{(\mathrm{up})}$ and $\mathbf{F}_{\mathrm{vv}}^{(\mathrm{up})}$ are diagonal matrices containing occupied and un-occupied orbital energies of the unperturbed system, respectively.

When the system is perturbed (initially or constantly), its KohnSham orbitals at a given time $t$ can be written as a unitary transformation of the orbitals of the unperturbed system

$$
\mathbf{C}(t)=\mathrm{U}(t) \mathbf{C}^{(\mathrm{up})}(t)
$$

where

$$
\mathbf{U}(t)=\exp \left(\begin{array}{cc}
0 & -\boldsymbol{\Theta}^{\dagger}(t) \\
\boldsymbol{\Theta}(t) & 0
\end{array}\right)
$$

depends on the current orbital rotation, $\boldsymbol{\Theta}(t)$, which is complex. Such a unitary transformation can be symbolically written as 9495

$$
\mathbf{U}(t)=\left(\begin{array}{cc}
\cos \sqrt{\boldsymbol{\Theta}^{\dagger}(t) \boldsymbol{\Theta}(t)} & -\frac{\sin \sqrt{\boldsymbol{\Theta}^{\dagger}(t) \boldsymbol{\Theta}(t)}}{\sqrt{\boldsymbol{\Theta}^{\dagger}(t) \boldsymbol{\Theta}(t)}} \boldsymbol{\Theta}^{\dagger}(t) \\
\boldsymbol{\Theta}(t) \frac{\sin \sqrt{\boldsymbol{\Theta}^{\dagger}(t) \boldsymbol{\Theta}(t)}}{\sqrt{\boldsymbol{\Theta}^{\dagger}(t) \boldsymbol{\Theta}(t)}} & \cos \sqrt{\boldsymbol{\Theta}(t) \boldsymbol{\Theta}^{\dagger}(t)}
\end{array}\right)
$$

The corresponding density matrix can be computed from the occupied orbitals

$$
\begin{aligned}
\mathbf{P}(t) & =\mathbf{C}_{\mathrm{o}}(t) \mathbf{C}_{\mathrm{o}}^{\dagger}(t) \\
& =\left(\begin{array}{cc}
\cos ^{2} \sqrt{\boldsymbol{\Theta}^{\dagger}(t) \boldsymbol{\Theta}(t)} & \frac{\sin \left[2 \sqrt{\boldsymbol{\Theta}^{\dagger}(t) \boldsymbol{\Theta}(t)}\right]}{2 \sqrt{\boldsymbol{\Theta}^{\dagger}(t) \boldsymbol{\Theta}(t)}} \boldsymbol{\Theta}^{\dagger}(t) \\
\Theta(t) \frac{\sin \left[2 \sqrt{\boldsymbol{\Theta}^{\dagger}(t) \boldsymbol{\Theta}(t)}\right]}{2 \sqrt{\boldsymbol{\Theta}^{\dagger}(t) \boldsymbol{\Theta}(t)}} & \sin ^{2} \sqrt{\boldsymbol{\Theta}(t) \boldsymbol{\Theta}^{\dagger}(t)}
\end{array}\right)
\end{aligned}
$$

In the limit of small orbital rotations, $\Theta(t) \rightarrow 0$, the unitary transformation and density matrix can by approximated by retaining only the first-order term,

$$
\begin{aligned}
\mathbf{U}(t) & =\left(\begin{array}{cc}
\mathbf{I}_{\mathrm{Oo}} & -\boldsymbol{\Theta}^{\dagger}(t) \\
\boldsymbol{\Theta}(t) & \mathbf{I}_{\mathrm{VV}}
\end{array}\right)+\mathscr{O}\left(\boldsymbol{\Theta}^{2}\right) \\
\mathbf{P}(t) & =\left(\begin{array}{cc}
\mathbf{I}_{\mathrm{OO}} & \boldsymbol{\Theta}^{\dagger}(t) \\
\boldsymbol{\Theta}(t) & \mathbf{0}
\end{array}\right)+\mathscr{O}\left(\boldsymbol{\Theta}^{2}\right)
\end{aligned}
$$

\section{A2. Initial Superposition Due to a Weak-field Perturbation}

In this work, the initial electronic state for RT-TDDFT calculations will be prepared by applying a weak static electric field of strength $\vec{f}=\left[f_{x}, f_{y}, f_{z}\right]$ at time zero $(t=0)$. The corresponding change to the core Hamiltonian is a linear combination of dipole moment matrices in Eq. 2

$$
(\delta h)_{a i}=\vec{f} \cdot \vec{\mu}_{a i}
$$

Such an electrostatic perturbation causes a unitary transformation in Eq. A6 from the unperturbed CMOs to the initial occupied MOs (of our RT-TDDFT calculations), which can be explicitly written as

$$
\psi_{i}(0)=\chi_{i}+\sum_{a} \chi_{a} \Theta_{a i}(0)
$$

These orbital rotations can be determined using the KS-DFT re- 
sponse kernel in Eq 10

$$
\begin{aligned}
\Theta_{a i}(0) & =-\sum_{b j}\left[(\mathbf{A}+\mathbf{B})^{-1}\right]_{a i, b j}(\delta h)_{b j} \\
& =-\sum_{m, b j} \frac{1}{\omega^{(m)}}\left(X_{a i}^{(m)}+Y_{a i}^{(m)}\right)\left(X_{b j}^{(m)}+Y_{b j}^{(m)}\right)(\delta h)_{b j} \\
& =-\sum_{m} \frac{\vec{f} \cdot \vec{\mu}^{(0, m)}}{\omega^{(m)}}\left(X_{a i}^{(m)}+Y_{a i}^{(m)}\right)
\end{aligned}
$$

where $\vec{\mu}^{(0, m)}$ is the transition dipole moment for the $m$-th excited state as defined in Eq. 7. Note that these orbital rotations have real elements, thus yielding all real Kohn-Sham orbitals at $t=0$ for all RT-TDDFT simulations in this work.

As mentioned above in Section 2.2, the "single-determinant" pseudowavefunction based on $\psi_{i}(0)$ can be interpreted as a superposition of electronic states in Eq 5 , which means

$$
\boldsymbol{\Theta}(0)=\sum_{m} a_{m}(0)(\mathbf{X}+\mathbf{Y})^{(m)}
$$

Comparing this to Eq. A10, the expansion coefficients are clearly

$$
a_{m}(0)=-\frac{\vec{f} \cdot \vec{\mu}^{(0, m)}}{\omega^{(m)}}
$$

One can multiply the identity matrix in Eq. 9 and the orbital rotations.

$$
\boldsymbol{\Theta}(0)=\sum_{m}(\mathbf{X}+\mathbf{Y})^{(m)}(\mathbf{X}-\mathbf{Y})^{(m)} \cdot \boldsymbol{\Theta}(0)
$$

Comparing it to Eq. A11. one arrives at an equivalent way to compute the superposition coefficients

$$
a_{m}(0)=\boldsymbol{\Theta}(0) \cdot(\mathbf{X}-\mathbf{Y})^{(m)}
$$

In this work, we focus on the weak-field limit, where the orbital rotations can be inferred from the vo-block of the initial density matrix (see Eq. A7). Accordingly, the expansion coefficients were approximated as

$$
\tilde{a}_{m}(0)=\mathbf{P}_{\mathrm{vo}}(0) \cdot(\mathbf{X}-\mathbf{Y})^{(m)}
$$

which are shown in Tables S1, S2, S3 to deviate by no more than $5 \%$ from $a_{m}(0)$ values computed using Eq. A12 for our three $\mathrm{Ag}_{4}-$ $\mathrm{N}_{2}$ test cases. In our work, the "superposition" results in Figs. 5, 6 and 7 were based on expansion coefficients computed using Eq. A15.

On the other hand, one can define a "conjugate" set of expansion coefficients,

$$
b_{m}(0)=\boldsymbol{\Theta}(0) \cdot(\mathbf{X}+\mathbf{Y})^{(m)}
$$

which can also produce the orbital rotations at $t=0$,

$$
\boldsymbol{\Theta}(0)=\sum_{m} b_{m}(0)(\mathbf{X}-\mathbf{Y})^{(m)}
$$

Combining Eqs. A11 and A17 one obtains

$$
\boldsymbol{\Theta}(0) \cdot \boldsymbol{\Theta}(0)=\sum_{a i} \Theta_{a i}(0)^{2}=\sum_{m} a_{m}(0) b_{m}(0)
$$

Therefore, the relative weight for the contribution from each excited state is

$$
p_{m}(0)=\frac{a_{m}(0) b_{m}(0)}{\boldsymbol{\Theta}(0) \cdot \boldsymbol{\Theta}(0)}
$$

\section{A3. RT-TDDFT Time-Evolution Following an Initial Weak-field Perturbation}

Beyond time zero, each electronic state component in Eq 5 is going to evolve independently in a field-free environment. For the $m$-th state, its $\mathbf{X}$ and $\mathbf{Y}$ amplitudes evolve with the frequency of $\omega_{m},{ }^{9697}$ leading to the following orbital rotation

$$
\begin{aligned}
\boldsymbol{\Theta}(t) & =\sum_{m} a_{m}(0)\left[\exp \left(-i \omega^{(m)} t\right) \mathbf{X}^{(m)}+\exp \left(i \omega^{(m)} t\right) \mathbf{Y}^{(m)}\right] \\
= & \sum_{m} a_{m}(0) \cos \left(\omega^{(m)} t\right)(\mathbf{X}+\mathbf{Y})^{(m)} \\
& -i \sum_{m} a_{m}(0) \sin \left(\omega^{(m)} t\right)(\mathbf{X}-\mathbf{Y})^{(m)}
\end{aligned}
$$

which can be plugged into Eqs. A6 and A7 to get the unitary transformation and density matrix at a given time.

The corresponding Fock matrix is

$$
\mathbf{F}(t)=\left(\begin{array}{cc}
\mathbf{F}_{\mathrm{oo}}^{(\mathrm{up})} & \mathbf{F}_{\mathrm{vo}}^{\mathrm{Re}, \mathrm{T}}-i \mathbf{F}_{\mathrm{vo}}^{\mathrm{Im}, \mathrm{T}} \\
\mathbf{F}_{\mathrm{vo}}^{\mathrm{Re}}+i \mathbf{F}_{\mathrm{vo}}^{\mathrm{Im}} & \mathbf{F}_{\mathrm{vv}}^{(\mathrm{up})}
\end{array}\right)+\mathscr{O}\left(\boldsymbol{\Theta}^{2}\right)
$$

whose $v o$ and $o v$ blocks can be easily shown to be

$$
\begin{aligned}
\mathbf{F}_{\mathrm{vo}}^{\mathrm{Re}}= & \sum_{m} a_{m}(0) \cos \left(\omega^{(m)} t\right)\left[\omega^{(m)}(\mathbf{X}-\mathbf{Y})^{(m)}\right. \\
& \left.-\mathbf{F}_{\mathrm{vV}}^{\text {(up })}(\mathbf{X}+\mathbf{Y})^{(m)}+(\mathbf{X}+\mathbf{Y})^{(m)} \mathbf{F}_{\mathrm{oO}}^{(\mathrm{up})}\right]+\mathscr{O}\left(\boldsymbol{\Theta}^{2}\right) \\
\mathbf{F}_{\mathrm{vo}}^{\mathrm{Im}} & =-\sum_{m} a_{m}(0) \sin \left(\omega^{(m)} t\right)\left[\omega^{(m)}(\mathbf{X}+\mathbf{Y})^{(m)}\right. \\
& \left.-\mathbf{F}_{\mathrm{vV}}^{\text {(up })}(\mathbf{X}-\mathbf{Y})^{(m)}+(\mathbf{X}-\mathbf{Y})^{(m)} \mathbf{F}_{\mathrm{oO}}^{(\text {up })}\right]+\mathscr{O}\left(\boldsymbol{\Theta}^{2}\right)
\end{aligned}
$$

The total dipole moment, $\vec{\mu}(t)$, is dependent only on the real part of the density matrix. It is expected to oscillate around $\vec{\mu}^{\text {(up) }}$ the dipole moment of the unperturbed system

$$
\vec{\mu}(t)=\vec{\mu}^{\text {(up) }}+2 \sum_{m} a_{m}(0) \cos \left(\omega^{(m)} t\right) \vec{\mu}^{(0, m)}
$$

This underlies the standard practice of Fourier transforming the dipole moments from a RT-TDDFT simulation within a weak field to produce the LR-TDDFT absorption spectrum of a system.

Furthermore, since the vo-block of the time-evolving density 
matrix in Eq. A7 are

$$
\begin{aligned}
\operatorname{Re}\left(\mathbf{P}_{\mathrm{vo}}(t)\right) & =\sum_{m} a_{m}(0) \cos \left(\omega^{(m)} t\right)(\mathbf{X}+\mathbf{Y})^{(m)} \\
\operatorname{Im}\left(\mathbf{P}_{\mathrm{vo}}(t)\right) & =-\sum_{m} a_{m}(0) \sin \left(\omega^{(m)} t\right)(\mathbf{X}-\mathbf{Y})^{(m)}
\end{aligned}
$$

their Fourier transforms lead to $(\mathbf{X}+\mathbf{Y})^{(m)}$ and $(\mathbf{X}-\mathbf{Y})^{(m)}$, whose combination produces LR-TDDFT amplitudes ( $\mathbf{X}$ and $\mathbf{Y}$ ) of each absorption peak. This constitutes the basis of "wavefunction" analysis ${ }^{109899}$ for excited states from RT-TDDFT simulations.

Given a set of orbital rotations, $\boldsymbol{\Theta}$, the energy change from the converged SCF solution is known to start from the second-order 97

$$
\begin{aligned}
& E(\boldsymbol{\Theta})-E(\boldsymbol{\Theta}=\mathbf{0}) \\
& =\frac{1}{2}\left[\boldsymbol{\Theta}^{*} \cdot \mathbf{A} \cdot \boldsymbol{\Theta}+\boldsymbol{\Theta} \cdot \mathbf{A} \cdot \boldsymbol{\Theta}^{*}+\boldsymbol{\Theta} \cdot \mathbf{B} \cdot \boldsymbol{\Theta}+\boldsymbol{\Theta}^{*} \cdot \mathbf{B} \cdot \boldsymbol{\Theta}^{*}\right]+\mathscr{O}\left(\boldsymbol{\Theta}^{3}\right) \\
& =\frac{1}{4}\left[\left(\boldsymbol{\Theta}+\boldsymbol{\Theta}^{*}\right) \cdot(\mathbf{A}+\mathbf{B}) \cdot\left(\boldsymbol{\Theta}+\boldsymbol{\Theta}^{*}\right)-\left(\boldsymbol{\Theta}-\boldsymbol{\Theta}^{*}\right) \cdot(\mathbf{A}-\mathbf{B}) \cdot\left(\boldsymbol{\Theta}-\boldsymbol{\Theta}^{*}\right)\right] \\
& \quad+\mathscr{O}\left(\boldsymbol{\Theta}^{3}\right)
\end{aligned}
$$

where $\mathbf{u} \cdot \mathbf{M} \cdot \mathbf{v}$ is a short-hand notation for $\sum_{a i, b j} u_{a i} M_{a i, b j} v_{b j}$ in different terms.

Plugging in the orbital rotations in Eq. A20 the second-order contribution to the RT-TDDFT energy in Eq. 14 is

$$
\begin{aligned}
& E(t)-E_{0}^{\text {(up) }}=\sum_{m, n} a_{m}(0) a_{n}(0) \\
& \times\left[\cos \left(\omega^{(m)} t\right) \cos \left(\omega^{(n)} t\right)(\mathbf{X}+\mathbf{Y})^{(m)} \cdot(\mathbf{A}+\mathbf{B}) \cdot(\mathbf{X}+\mathbf{Y})^{(n)}\right. \\
& \left.\quad+\sin \left(\omega^{(m)} t\right) \sin \left(\omega^{(n)} t\right)(\mathbf{X}-\mathbf{Y})^{(m)} \cdot(\mathbf{A}-\mathbf{B}) \cdot(\mathbf{X}-\mathbf{Y})^{(n)}\right] \\
& =\sum_{m, n} a_{m}(0) a_{n}(0)\left[\cos \left(\omega^{(m)} t\right) \cos \left(\omega^{(n)} t\right) \omega_{m} \delta_{m n}\right. \\
& \left.\quad+\sin \left(\omega^{(m)} t\right) \sin \left(\omega^{(n)} t\right) \omega_{m} \delta_{m n}\right] \\
& =\sum_{m} a_{m}^{2}(0) \omega_{m}
\end{aligned}
$$

which clearly remains constant during a RT-TDDFT simulation. This, together with higher-order terms that must also remain constant, ensures that RT-TDDFT energy is conserved in a fieldfree environment.

\section{References}

1 C. Yam, S. Yokojima and G. Chen, Linear-Scaling TimeDependent Density-Functional Theory, Phys. Rev. B, 2003, 68, 153105.

2 X. Li, S. M. Smith, A. N. Markevitch, D. A. Romanov, R. J. Levis and H. B. Schlegel, A Time-Dependent Hartree-Fock Approach for Studying the Electronic Optical Response of Molecules in Intense Fields, Phys. Chem. Chem. Phys., 2005, 7, 233-239.

3 C.-L. Cheng, J. S. Evans and T. Van Voorhis, Simulating Molecular Conductance Using Real-Time Density Functional Theory,
Phys. Rev. B, 2006, 74, 155112.

4 J. Sun, J. Song, Y. Zhao and W.-Z. Liang, Real-Time Propagation of the Reduced One-electron Density Matrix in Atomcentered Gaussian Orbitals: Application to Absorption Spectra of Silicon Clusters, J. Chem. Phys., 2007, 127, 234107.

5 M. R. Provorse and C. M. Isborn, Electron Dynamics with Real-Time Time-Dependent Density Functional Theory, Int. J. Quantum Chem., 2016, 116, 739-749.

6 J. J. Goings, P. J. Lestrange and X. Li, Real-Time TimeDependent Electronic Structure Theory, WIRES Comput. Mol. Sci., 2018, 8, e1341.

7 S. Tussupbayev, N. Govind, K. Lopata and C. J. Cramer, Comparison of Real-Time and Linear-Response Time-Dependent Density Functional Theories for Molecular Chromophores Ranging from Sparse to High Densities of States, J. Chem. Theory Comput., 2015, 11, 1102-1109.

8 K. Lopata, B. E. Van Kuiken, M. Khalil and N. Govind, LinearResponse and Real-Time Time-Dependent Density Functional Theory Studies of Core-Level Near-Edge X-Ray Absorption, $J$. Chem. Theory Comput., 2012, 8, 3284-3292.

9 M. Kadek, L. Konecny, B. Gao, M. Repisky and K. Ruud, XRay Absorption Resonances Near L 2,3 -Edges From Real-Time Propagation of the Dirac-Kohn-Sham Density Matrix, Phys. Chem. Chem. Phys., 2015, 17, 22566-22570.

10 A. Bruner, D. LaMaster and K. Lopata, Accelerated Broadband Spectra Using Transition Dipole Decomposition and Pade Approximants, J. Chem. Theory Comput., 2016, 12, 3741-3750.

11 S. M. Smith, X. Li, A. N. Markevitch, D. A. Romanov, R. J. Levis and H. B. Schlegel, A Numerical Simulation of Nonadiabatic Electron Excitation in the Strong Field Regime: Linear Polyenes, J. Phys. Chem. A, 2005, 109, 5176-5185.

12 S. M. Smith, X. Li, A. N. Markevitch, D. A. Romanov, R. J. Levis and H. B. Schlegel, Numerical Simulation of Nonadiabatic Electron Excitation in the Strong Field Regime. 2. Linear Polyene Cations, J. Phys. Chem. A, 2005, 109, 10527-10534.

13 S. M. Smith, X. Li, A. Markevitch, D. Romanov, R. J. Levis and H. B. Schlegel, Numerical Simulation of Nonadiabatic Electron Excitation in the Strong-Field Regime. 3. Polyacene Neutrals and Cations, J. Phys. Chem. A, 2007, 111, 6920-6932.

14 H. B. Schlegel, S. M. Smith and X. Li, Electronic Optical Response of Molecules in Intense Fields: Comparison of TD-HF, TD-CIS, and TD-CIS(D) approaches, J. Chem. Phys., 2007, 126, 244110.

15 F. Ding, E. B. Guidez, C. M. Aikens and X. Li, Quantum Coherent Plasmon in Silver Nanowires: A Real-Time TDDFT Study, J. Chem. Phys., 2014, 140, 244705.

16 R. D. Senanayake, D. B. Lingerfelt, G. U. Kuda-Singappulige, X. Li and C. M. Aikens, Real-Time TDDFT Investigation of Optical Absorption in Gold Nanowires, J. Phys. Chem. C, 2019, 123, 14734-14745.

17 T. P. Rossi, M. Kuisma, M. J. Puska, R. M. Nieminen and P. Erhart, Kohn-Sham Decomposition in Real-Time Time-Dependent Density-Functional Theory: An Efficient Tool for Analyzing Plasmonic Excitations, J. Chem. Theory Comput., 2017, 13, 4779-4790. 
$18 \mathrm{H}$. Eshuis and T. van Voorhis, The Influence of Initial Conditions on Charge Transfer Dynamics, Phys. Chem. Chem. Phys., 2009, 11, 10293.

19 C. M. Isborn and X. Li, Singlet-Triplet Transitions in Real-Time Time-Dependent Hartree-Fock/Density Functional Theory, $J$. Chem. Theory Comput., 2009, 5, 2415-2419.

20 J. Pilme, E. Luppi, J. Berges, C. Houee-Levin and A. de la Lande, Topological Analyses of Time-Dependent Electronic Structures: Application to Electron-Transfers in Methionine Enkephalin, J. Mol. Model., 2014, 20, 2368.

21 A. Parise, A. Alvarez-Ibarra, X. Wu, X. Zhao, J. Pilme and A. de la Lande, Quantum Chemical Topology of the Electron Localization Function in the Field of Attosecond Electron Dynamics, J. Phys. Chem. Lett., 2018, 9, 844-850.

22 F. Wang, C. Y. Yam and G. Chen, Time-Dependent DensityFunctional Theory/Localized Density Matrix Method for Dynamic Hyperpolarizability, J. Chem. Phys., 2007, 126, 244102.

23 Y. Takimoto, F. D. Vila and J. J. Rehr, Real-Time TimeDependent Density Functional Theory Approach for FrequencyDependent Nonlinear Optical Response in Photonic Molecules, J. Chem. Phys., 2007, 127, 154114.

24 J. J. Goings and X. Li, An Atomic Orbital Based Real-Time Time-Dependent Density Functional Theory for Computing Electronic Circular Dichroism Band Spectra, J. Chem. Phys., 2016, 144, 234102.

25 X. Li and J. C. Tully, Ab initio Time-Resolved Density Functional Theory for Lifetimes of Excited Adsorbate States at Metal Surfaces, Chem. Phys. Lett., 2007, 439, 199-203.

26 Z. Guo, W. Liang, Y. Zhao and G. Chen, Real-Time Propagation of the Reduced One-Electron Density Matrix in Atom-Centered Orbitals: Application to Electron Injection Dynamics in DyeSensitized $\mathrm{TiO}_{2}$ Clusters, J. Phys. Chem. C, 2008, 112, 1665516662.

27 J. Jornet-Somoza and I. Lebedeva, Real-Time Propagation TDDFT and Density Analysis for Exciton Coupling Calculations in Large Systems, J. Chem. Theory Comput., 2019, 15, 37433754.

28 A. de la Lande, A. Alvarez-Ibarra, K. Hasnaoui, F. Cailliez, X. Wu, T. Mineva, J. Cuny, P. Calaminici, L. Lopez-Sosa, G. Geudtner, I. Navizet, C. Garcia Iriepa, D. R. Salahub and A. M. Koster, Molecular Simulations with in-deMon2k QM/MM, a Tutorial-Review, Molecules, 2019, 24, 1653.

29 A. Alvarez-Ibarra, A. Parise, K. Hasnaoui and A. de la Lande, The Physical Stage of Radiolysis of Solvated DNA by HighEnergy-Transfer Particles: Insights From New First Principles Simulations, Phys. Chem. Chem. Phys., 2020, 22, 7747-7758.

30 B. Peng, D. B. Lingerfelt, F. Ding, C. M. Aikens and X. Li, RealTime TDDFT Studies of Exciton Decay and Transfer in Silver Nanowire Arrays, J. Phys. Chem. C, 2015, 119, 6421-6427.

31 O. A. Hull, D. B. Lingerfelt, X. Li and C. M. Aikens, Electronic Structure and Nonadiabatic Dynamics of Atomic Silver Nanowire- $\mathrm{N}_{2}$ Systems, J. Phys. Chem., Revisions Submitted.

32 U. Aslam, V. G. Rao, S. Chavez and S. Linic, Catalytic Conversion of Solar to Chemical Energy on Plasmonic Metal Manostructures, Nat. Catal., 2018, 1, 656-665.
33 S. Mukherjee, F. Libisch, N. Large, O. Neumann, L. V. Brown, J. Cheng, J. B. Lassiter, E. A. Carter, P. Nordlander and N. J. Halas, Hot Electrons Do the Impossible: Plasmon-Induced Dissociation of $\mathrm{H}_{2}$ on Au, Nano Lett., 2013, 13, 240-247.

34 L. Zhou, C. Zhang, M. J. McClain, A. Manjavacas, C. M. Krauter, S. Tian, F. Berg, H. O. Everitt, E. A. Carter, P. Nordlander and N. J. Halas, Aluminum Nanocrystals as a Plasmonic Photocatalyst for Hydrogen Dissociation, Nano Lett., 2016, 16, 1478-1484.

35 C. Zhang, H. Zhao, L. Zhou, A. E. Schlather, L. Dong, M. J. McClain, D. F. Swearer, P. Nordlander and N. J. Halas, Al-Pd Nanodisk Heterodimers as Antenna-Reactor Photocatalysts, Nano Lett., 2016, 16, 6677-6682.

36 D. F. Swearer, H. Zhao, L. Zhou, C. Zhang, H. Robatjazi, J. M. P. Martirez, C. M. Krauter, S. Yazdi, M. J. McClain, E. Ringe, E. A. Carter, P. Nordlander and N. J. Halas, Heterometallic AntennaReactor Complexes for Photocatalysis, Proc. Natl. Acad. Sci. USA, 2016, 113, 8916-8920.

37 P. Christopher, H. Xin and S. Linic, Visible-Light-Enhanced Catalytic Oxidation Reactions on Plasmonic Silver Nanostructures, Nat. Chem., 2011, 3, 467-472.

38 P. Christopher, H. Xin, A. Marimuthu and S. Linic, Singular Characteristics and Unique Chemical Bond Activation Mechanisms of Photocatalytic Reactions on Plasmonic Nanostructures, Nat. Mat., 2012, 11, 1044-1050.

39 B. Seemala, A. J. Therrien, M. Lou, K. Li, J. P. Finzel, J. Qi, P. Nordlander and P. Christopher, Plasmon-Mediated Catalytic O2 Dissociation on Ag Nanostructures: Hot Electrons or Near Fields?, ACS Energy Letters, 2019, 4, 1803-1809.

40 J. M. P. Martirez and E. A. Carter, Excited-State $\mathrm{N}_{2}$ Dissociation Pathway on Fe-Functionalized Au, J. Am. Chem. Soc., 2017, 139, 4390-4398.

41 J. M. P. Martirez and E. A. Carter, Prediction of a LowTemperature $\mathrm{N}_{2}$ Dissociation Catalyst Exploiting Near-IR-toVisible Light Nanoplasmonics, Sci. Adv., 2017, 3, eaao4710.

42 L. Zhou, D. F. Swearer, C. Zhang, H. Robatjazi, H. Zhao, L. Henderson, L. Dong, P. Christopher, E. A. Carter, P. Nordlander and N. J. Halas, Quantifying Hot Carrier and Thermal Contributions in Plasmonic Photocatalysis, Science, 2018, 362, 69-72.

43 E. C. M. Barbosa, J. L. Fiorio, T. Mou, B. Wang, L. M. Rossi and P. H. C. Camargo, Reaction Pathway Dependence in Plasmonic Catalysis: Hydrogenation as a Model Molecular Transformation, Chem. Eur. J., 2018, 24, 12330-12339.

44 J. Quiroz, E. C. M. Barbosa, T. P. Araujo, J. L. Fiorio, Y.-C. Wang, Y.-C. Zou, T. Mou, T. V. Alves, D. C. de Oliveira, B. Wang, S. J. Haigh, L. M. Rossi and P. H. C. Camargo, Controlling Reaction Selectivity over Hybrid Plasmonic Nanocatalysts, Nano Lett., 2018, 18, 7289-7297.

45 H. Nakai, Energy density analysis with Kohn-Sham orbitals, Chem. Phys. Lett., 2002, 363, 73-79.

46 Y. Imamura, A. Takahashi and H. Nakai, Grid-Based Energy Density Analysis: Implementation and assessment, J. Chem. Phys., 2007, 126, 034103. 
47 H. Nakai, H. Ohashi, Y. Imamura and Y. Kikuchi, Bond Energy Analysis Revisited and Designed Toward a Rigorous Methodology, J. Chem. Phys., 2011, 135, 124105.

48 M. E. Casida, in Recent Advances in Density Functional Methods Part I., World Scientific, 1995, p. 155.

49 R. Bauernschmitt and R. Ahlrichs, Treatment of Electronic Excitations within the Adiabatic Approximation of Time Dependent Density Functional Theory, Chem. Phys. Lett., 1996, 256, 454-464.

50 S. Hirata and M. Head-Gordon, Time-Dependent Density Functional Theory for Radicals, Chem. Phys. Lett., 1999, 302, 375382.

51 A. Dreuw and M. Head-Gordon, Single-Reference ab Initio Methods for the Calculation of Excited States of Large Molecules, Chem. Rev., 2005, 105, 4009-4037.

52 M. Casida and M. Huix-Rotllant, Progress in Time-Dependent Density-Functional Theory, Ann. Rev. Phys. Chem., 2012, 63, 287-323.

53 Z. Pei, J. Yang, J. Deng, Y. Mao, Q. Wu, Z. Yang, B. Wang, C. M. Aikens, W. Liang and Y. Shao, Can We See the Energy Density? II. Insights from Linear-Response Time-Dependent Density Functional Theory Calculations, Under Review.

54 F. Furche, On the Density Matrix Based Approach to TimeDependent Density Functional Response Theory, J. Chem. Phys., 2001, 114, 5982-5992.

55 K. Lopata and N. Govind, Modeling Fast Electron Dynamics with Real-Time Time-Dependent Density Functional Theory: Application to Small Molecules and Chromophores, J. Chem. Theory Comput., 2011, 7, 1344-1355.

56 J. Tao, J. P. Perdew, V. N. Staroverov and G. E. Scuseria, Climbing the Density Functional Ladder: Nonempirical MetaGeneralized Gradient Approximation Designed for Molecules and Solids, Phys. Rev. Lett., 2003, 91, 146401.

57 Y. Zhao and D. G. Truhlar, The M06 Suite of Density Functionals for Main Group Thermochemistry, Thermochemical Kinetics, Noncovalent Interactions, Excited states, and Transition Elements: Two New Functionals and Systematic Testing of Four M06-Class Functionals and 12 Other Functionals, Theor. Chem. Acc., 2008, 120, 215-241.

58 A. D. Becke, Real-Space Post-Hartree-Fock Correlation Models, J. Chem. Phys., 2005, 122, 064101.

59 E. Proynov, Y. Shao and J. Kong, Efficient Self-Consistent DFT Calculation of Nondynamic Correlation Based on the B05 Method, Chem. Phys. Lett., 2010, 493, 381-385.

60 E. Proynov, F. Liu, Y. Shao and J. Kong, Improved SelfConsistent and Resolution-of-Identity Approximated Becke'05 Density Functional Model of Nondynamic Electron Correlation, J. Chem. Phys., 2012, 136, 034102.

61 Q. Sun, T. C. Berkelbach, N. S. Blunt, G. H. Booth, S. Guo, Z. Li, J. Liu, J. D. McClain, E. R. Sayfutyarova, S. Sharma, S. Wouters and G. K. Chan, PySCF: the Python-Based Simulations of Chemistry Framework, WIREs Comput. Mol. Sci., 2018, 8, e1340.

62 J. Rezac and A. de la Lande, Robust, Basis-Set Independent Method for the Evaluation of Charge-Transfer Energy in Non- covalent Complexes, J. Chem. Theory Comput., 2015, 11, 528537.

63 A. D. Becke, A Multicenter Numerical Integration Scheme for Polyatomic Molecules, J. Chem. Phys., 1988, 88, 2547-2553.

64 J. P. Perdew, K. Burke and M. Ernzerhof, Generalized Gradient Approximation Made Simple, Phys. Rev. Lett., 1996, 77, 38653868.

65 C. Adamo and V. Barone, Toward Reliable Density Functional Methods Without Adjustable Parameters: The PBE0 Model, J. Chem. Phys., 1999, 110, 6158-6170.

66 A. D. Becke, Density-functional Exchange-Energy Approximation with Correct Asymptotic Behavior, Phys. Rev. A, 1988, 38, 3098-3100.

67 A. D. Becke, A New Mixing of Hartree-Fock and Local DensityFunctional Theories, J. Chem. Phys., 1993, 98, 1372-1377.

68 C. Lee, W. Yang and R. G. Parr, Development of the ColleSalvetti Correlation-Energy Formula into a Functional of the Electron Density, Phys. Rev. B, 1988, 37, 785-789.

69 J.-D. Chai and M. Head-Gordon, Long-Range Corrected Hybrid Density Functionals with Damped Atom-Atom Dispersion Corrections, Phys. Chem. Chem. Phys., 2008, 10, 6615.

70 D. Andrae, U. Haeussermann, M. Dolg, H. Stoll and H. Preuss, Energy-adjusted ab initio pseudopotentials for the second and third row transition elements, Theor. Chim. Acta, 1990, 77, 123-141.

71 B. B. Dale, R. D. Senanayake and C. M. Aikens, Density Functional Theory Investigation of the Interactions of Silver Nanoclusters with Guanine, APL Materials, 2017, 5, 053102.

72 A. Castro, M. A. L. Marques and A. Rubio, Propagators for the Time-Dependent Kohn-Sham Equations, J. Chem. Phys., 2004, 121, 3425-3433.

73 F. Casas and A. Iserles, Explicit Magnus Expansions for Nonlinear Equations, J. Phys A: Math. Gen., 2006, 39, 5445-5461.

74 T. S. Nguyen and J. Parkhill, Nonadiabatic Dynamics for Electrons at Second-Order: Real-Time TDDFT and OSCF2, J. Chem. Theory Comput., 2015, 11, 2918-2924.

75 A. Gomez Pueyo, M. A. L. Marques, A. Rubio and A. Castro, Propagators for the Time-Dependent Kohn-Sham Equations: Multistep, Runge-Kutta, Exponential Runge-Kutta, and Commutator Free Magnus Methods, J. Chem. Theory Comput., 2018, 14, 3040-3052.

76 Y. Zhu and J. M. Herbert, Self-consistent Predictor/Corrector Algorithms for Stable and Efficient Integration of the TimeDependent Kohn-Sham Equation, J. Chem. Phys., 2018, 148, 044117.

77 D. B. Williams-Young, A. Petrone, S. Sun, T. F. Stetina, P. Lestrange, C. E. Hoyer, D. R. Nascimento, L. Koulias, A. Wildman, J. Kasper, J. J. Goings, F. Ding, A. E. DePrince, E. F. Valeev and X. Li, The Chronus Quantum Software Package, WIRES Comput. Mol. Sci., 2020, 10, In Press.

78 M. Dolg and X. Cao, Relativistic Pseudopotentials: Their Development and Scope of Applications, Chem. Rev., 2012, 112, 403-480.

79 S. C. McKenzie, E. Epifanovsky, G. M. J. Barca, A. T. B. Gilbert 
and P. M. W. Gill, Efficient Method for Calculating Effective Core Potential Integrals, J. Phys. Chem. A, 2018, 122, 30663075.

80 M. Head-Gordon, A. M. Grana, D. Maurice and C. A. White, Analysis of Electronic Transitions as the Difference of Electron Attachment and Detachment Densities, J. Phys. Chem., 1995, 99, 14261-14270.

81 J. E. Subotnik, A. Jain, B. Landry, A. Petit, W. Ouyang and N. Bellonzi, Understanding the Surface Hopping View of Electronic Transitions and Decoherence, Annu. Rev. Phys. Chem., 2016, 67, 387-417.

82 R. Crespo-Otero and M. Barbatti, Recent Advances and Perspectives on Nonadiabatic Mixed Quantum-Classical Dynamics, Chem. Rev., 2018, 118, 7026-7068.

83 X. Li, J. C. Tully, H. B. Schlegel and M. J. Frisch, Ab initio Ehrenfest Dynamics, J. Chem. Phys., 2005, 123, 084106.

84 C. M. Isborn, X. Li and J. C. Tully, Time-Dependent Density Functional Theory Ehrenfest Dynamics: Collisions Between Atomic Oxygen and Graphite Clusters, J. Chem. Phys., 2007, 126, 134307.

85 F. Wang, C. Y. Yam, L. Hu and G. Chen, Time-Dependent Density Functional Theory Based Ehrenfest Dynamics, J. Chem. Phys., 2011, 135, 044126.

86 O. Vahtras, J. Almlof and M. Feyereisen, Integral Approximations for LCAO-SCF Calculations, Chem. Phys. Lett., 1993, 213, 514-518.

87 K. Eichkorn, O. Treutler, H. Ohm, M. Haser and R. Ahlrichs, Auxiliary Basis Sets to Approximate Coulomb Potentials, Chem. Phys. Lett., 1995, 240, 283-290.

88 K. Eichkorn, F. Weigend, O. Treutler and R. Ahlrichs, Auxiliary Basis Sets for Main Row Atoms and Transition Metals and Their Use to Approximate Coulomb Potentials, Theor. Chem. Acta, 1997, 97, 119-124.

89 B. Dunlap, Robust and Variational Fitting: Removing the FourCenter Integrals from Center Stage in Quantum Chemistry, $J$. Mol. Struct. THEOCHEM, 2000, 529, 37-40.

90 B. Dunlap, Robust Variational Fitting: Gaspar's Variational Ex- change Can Accurately Be Treated Analytically, J. Mol. Struct. THEOCHEM, 2000, 501-502, 221-228.

91 Y. Jung, A. Sodt, P. M. W. Gill and M. Head-Gordon, Auxiliary Basis Expansions for Large-Scale Electronic Structure Calculations, Proc. Natl. Acad. Sci. USA, 2005, 102, 6692-6697.

92 D. S. Hollman, H. F. Schaefer and E. F. Valeev, Semi-Exact Concentric Atomic Density Fitting: Reduced Cost and Increased Accuracy Compared to Standard Density Fitting, J. Chem. Phys., 2014, 140, 064109.

93 H. F. Schurkus, A. Luenser and C. Ochsenfeld, Almost ErrorFree Resolution-of-the-Identity Correlation Methods by Null Space Removal of the Particle-Hole Interactions, J. Chem. Phys., 2017, 146, 211106.

94 J. Hutter, M. Parrinello and S. Vogel, Exponential Transformation of Molecular Orbitals, J. Chem. Phys., 1994, 101, 38623865.

95 T. Van Voorhis and M. Head-Gordon, A Geometric Approach to Direct Minimization, Mol. Phys., 2002, 100, 1713-1721.

96 R. Send and F. Furche, First-Order Nonadiabatic Couplings From Time-Dependent Hybrid Density Functional Response Theory: Consistent Formalism, Implementation, and Performance, J. Chem. Phys., 2010, 132, 044107.

97 T. Ziegler, M. Krykunov and J. Autschbach, Derivation of the RPA (Random Phase Approximation) Equation of ATDDFT (Adiabatic Time Dependent Density Functional Ground State Response Theory) from an Excited State Variational Approach Based on the Ground State Functional, J. Chem. Theory Comput., 2014, 10, 3980-3986.

98 M. Repisky, L. Konecny, M. Kadek, S. Komorovsky, O. L. Malkin, V. G. Malkin and K. Ruud, Excitation Energies from Real-Time Propagation of the Four-Component Dirac-Kohn-Sham Equation, J. Chem. Theory Comput., 2015, 11, 980-991.

99 S. Ghosh, J. C. Asher, L. Gagliardi, C. J. Cramer and N. Govind, A Semiempirical Effective Hamiltonian Based Approach for Analyzing Excited State Wave Functions and Computing Excited State Absorption Spectra Using Real-Time Dynamics, J. Chem. Phys., 2019, 150, 104103. 


\title{
Can We See the Energy Densities? I. Insights from Real-Time Time-Dependent Density Functional Theory Simulations. Electronic Supplementary Information
}

\author{
Junjie Yang, ${ }^{a, \#}$ Zheng Pei ${ }^{b}, \#$, Jingheng Deng ${ }^{a}$, Yuezhi $\mathrm{Mao}^{c}$, Qin $\mathrm{Wu}^{d}$, \\ Zhibo Yang ${ }^{a}$, Bin Wang ${ }^{e}$, Christine M. Aikens ${ }^{f}$, Wanzhen Liang* ${ }^{* b}$, and Yihan Shao*a
}

August 2, 2020

${ }^{a}$ Department of Chemistry and Biochemistry, University of Oklahoma, 101 Stephenson Pkwy, Norman, OK 73019, United States. E-mail: yihan.shao@ou.edu

$b$ State Key Laboratory of Physical Chemistry of Solid Surfaces, Collaborative Innovation Center of Chemistry for Energy Materials, Fujian Provincial Key Laboratory of Theoretical and Computational Chemistry, and Department of Chemistry, College of Chemistry and Chemical Engineering, Xiamen University, Xiamen 361005, P. R. China. E-mail: liangwz@xmu.edu.cn

c Department of Chemistry, Stanford University, Stanford, CA 94305, United States.

${ }^{d}$ Center for Functional Nanomaterials, Brookhaven National Laboratory, Upton, NY 11973, United States.

e Center for Interfacial Reaction Engineering and School of Chemical, Biological, and Materials Engineering, Gallogly College of Engineering, University of Oklahoma, Norman, OK 73019, United States.

$f$ Department of Chemistry, Kansas State University, Manhattan, KS 66506, United States.

\# These two authors contributed equally to this work. 


\section{Propagators for RT-TDDFT simulations}

\subsection{Different RT-TDDFT Propagation Schemes}

Here a brief summary is provided on several RT-TDDFT propagation schemes implemented in this work.

\section{a) MMUT}

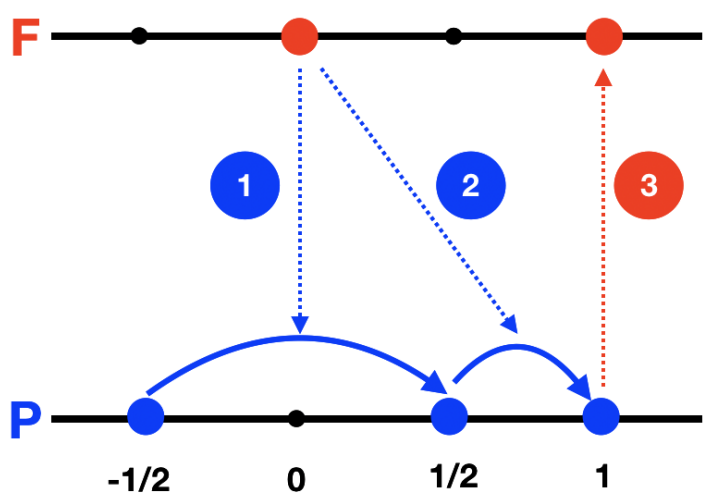

c) EP-PC

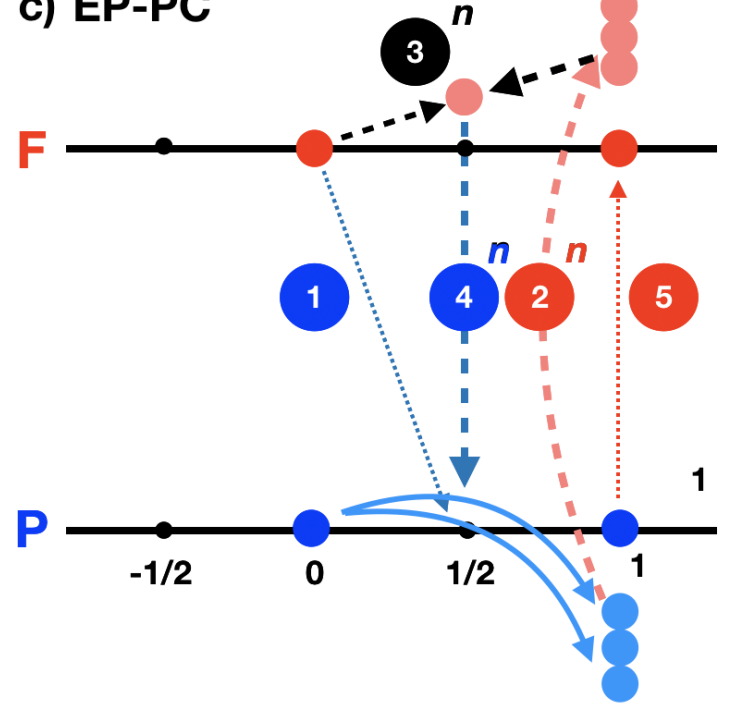

b) AMUT-k, RKMK-Heun

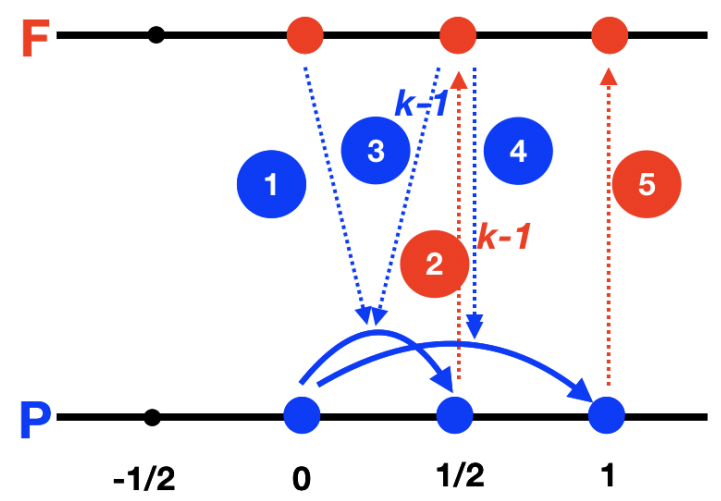

d) LFLP-PC

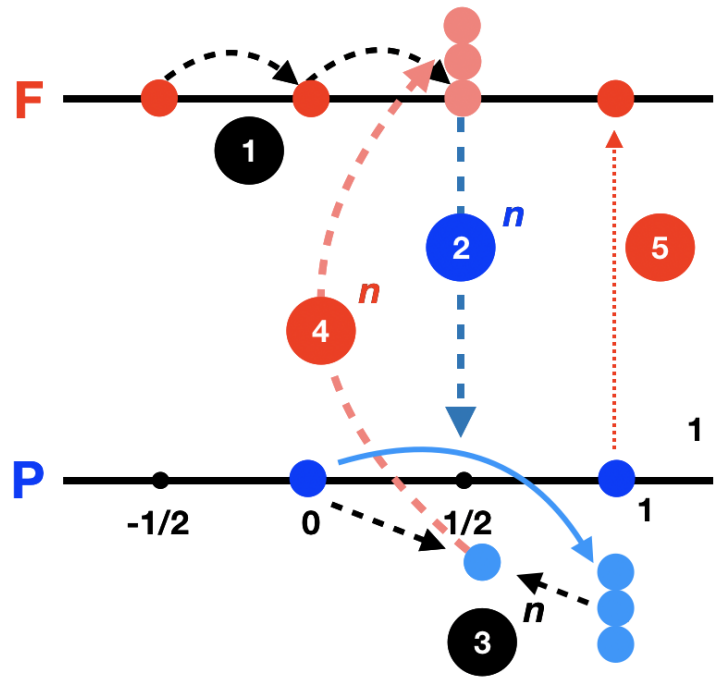

Figure S1: Different RT-TDDFT propagation schemes tested in the work. 


\subsubsection{Modified Midpoint Unitary Transformation (MMUT)}

The MMUT scheme was proposed by Li et al [Phys. Chem. Chem. Phys. 7, 233 (2005)]. Here is the workflow of our implementation.

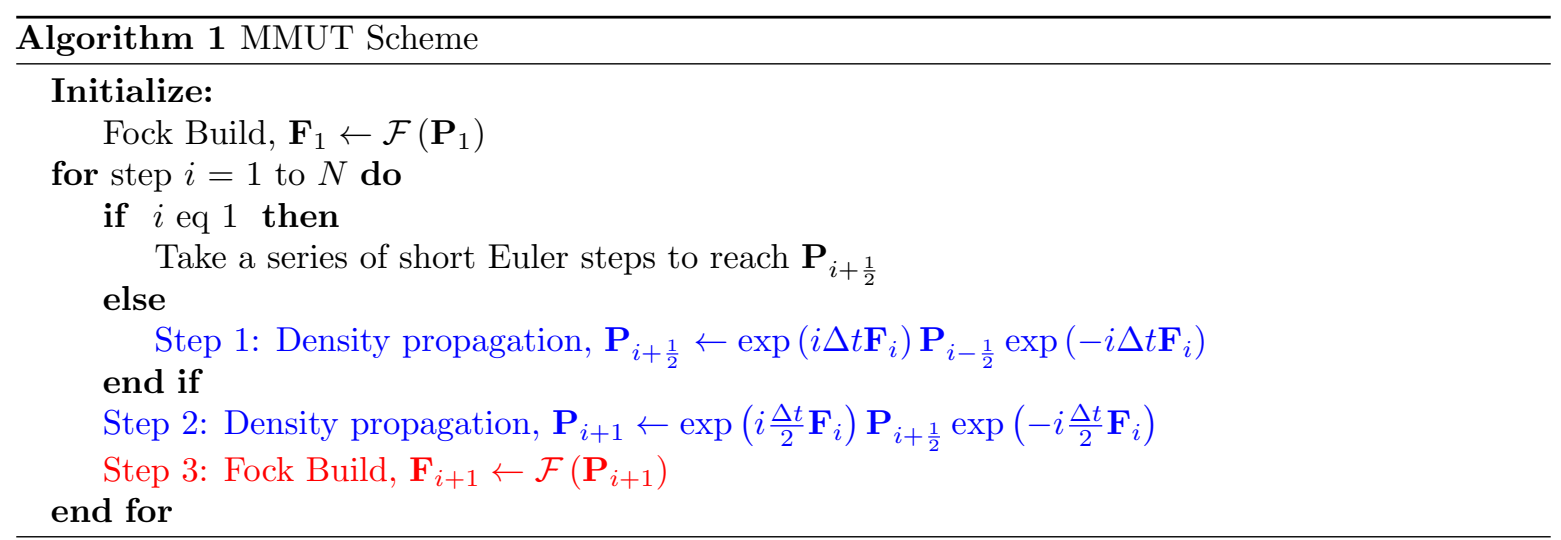

\subsubsection{Approximate Midpoint Unitary Transformation (AMUT- $k$ )}

Below is the workflow of our implementation of AMUT- $k$ schemes. When $k=1$, it reduces to the Runge-Kutta-Munthe-Kass-Heun (RKMK-Heun) scheme [see Eq. 20 of Casas and Iserles, J. Phys. A: Math. Gen. 39, 5445 (2006).]

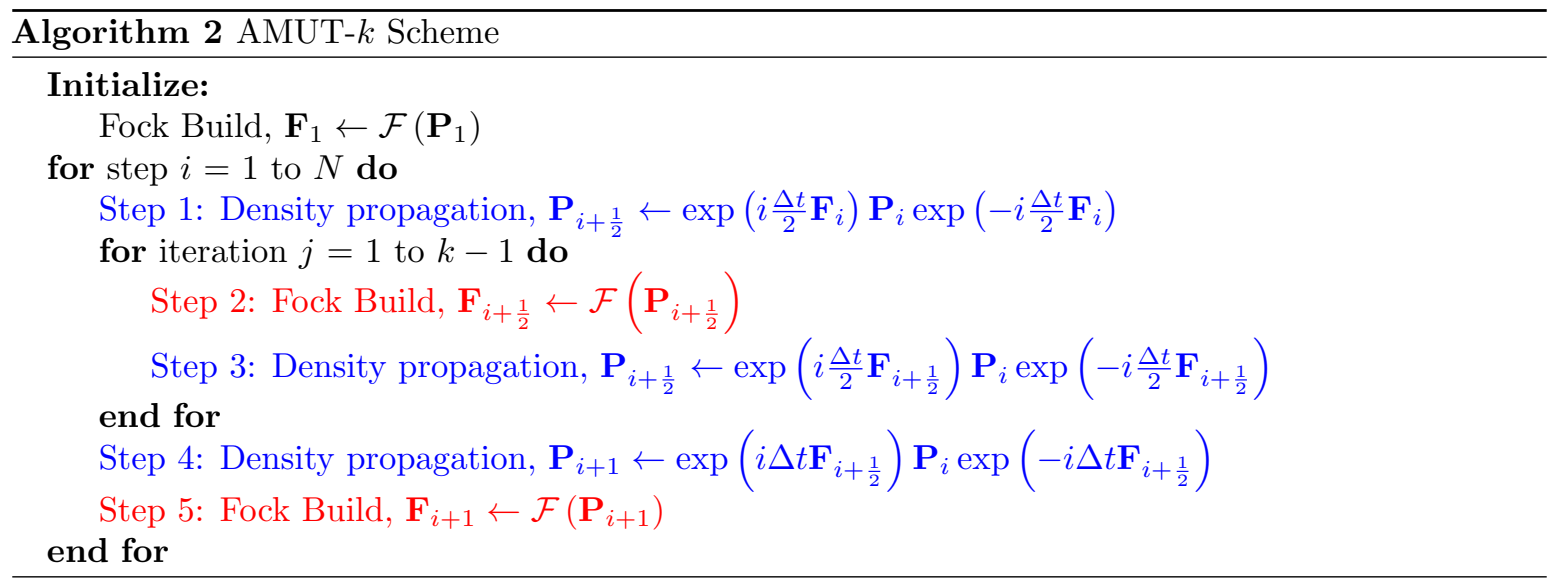

\subsubsection{Exponential Density Predictor-Corrector (EP-PC) and Linear Fock Linear Density Predictor-Corrector (LFLP-PC)}

The EP-PC and LFLP-PC schemes were proposed by Zhu and Herbert [J. Chem. Phys. 148, 044117 (2018)]. Here is the workflow of our implementations. 

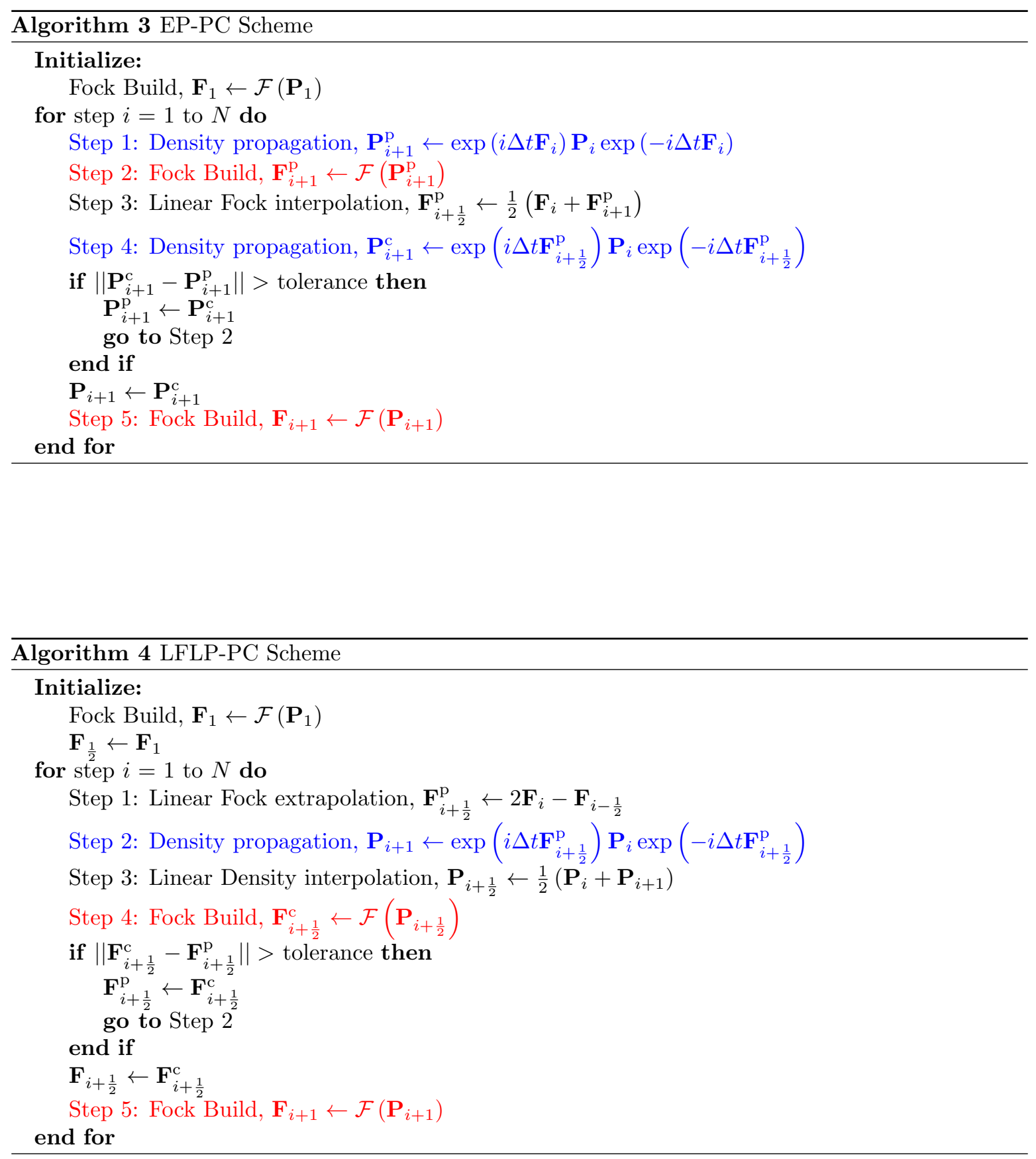


\subsection{Energy Conservation in RT-TDDFT Simulations}
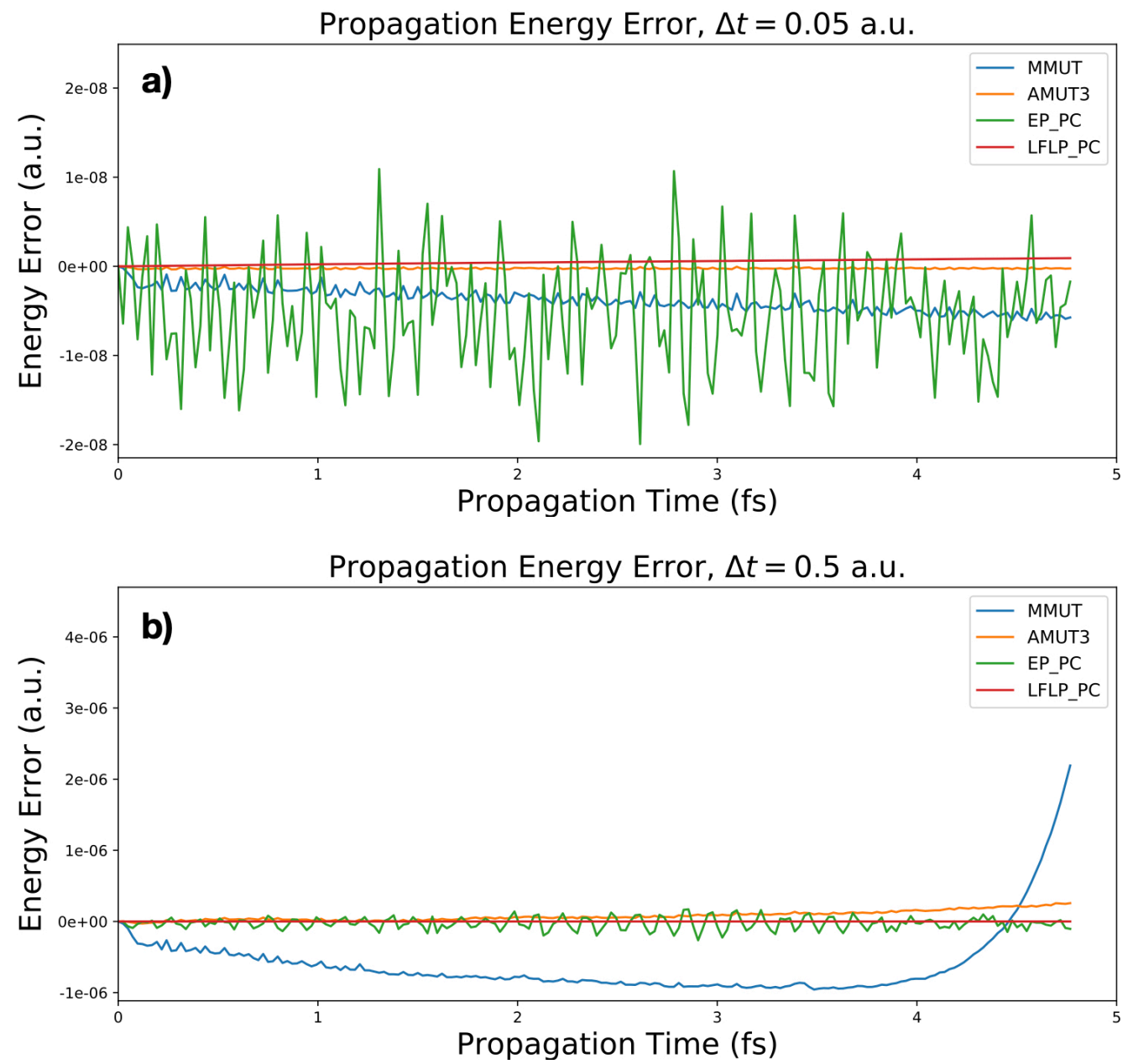

Figure S2: Energy propagation errors (in a.u.) from RT-TDDFT calculations on the stacked-long $\mathrm{Ag}_{4}-\mathrm{N}_{2}$ complex. The errors are computed with reference to the energy at $t=0$. Results with a time step of 0.05 and 0.5 a.u. are shown in panels $a$ and $b$, respectively. PBE0 functional and 6-31G(d) basis set were used. 


\section{Fluctuation of Energy Components Along RT-TDDFT Tra- jectories}

The fluctuations in different energy components along RT-TDDFT trajectories are shown below for three $\mathrm{Ag}_{4}-\mathrm{N}_{2}$ complexes. PBE0 functional, LFLP-PC propagator, and a 0.5 a.u. time step were used in these simulations. Stuttgart effective core potential and basis set were employed for silver atoms, and $6-31 \mathrm{G}(\mathrm{d})$ basis functions were used on nitrogen atoms.
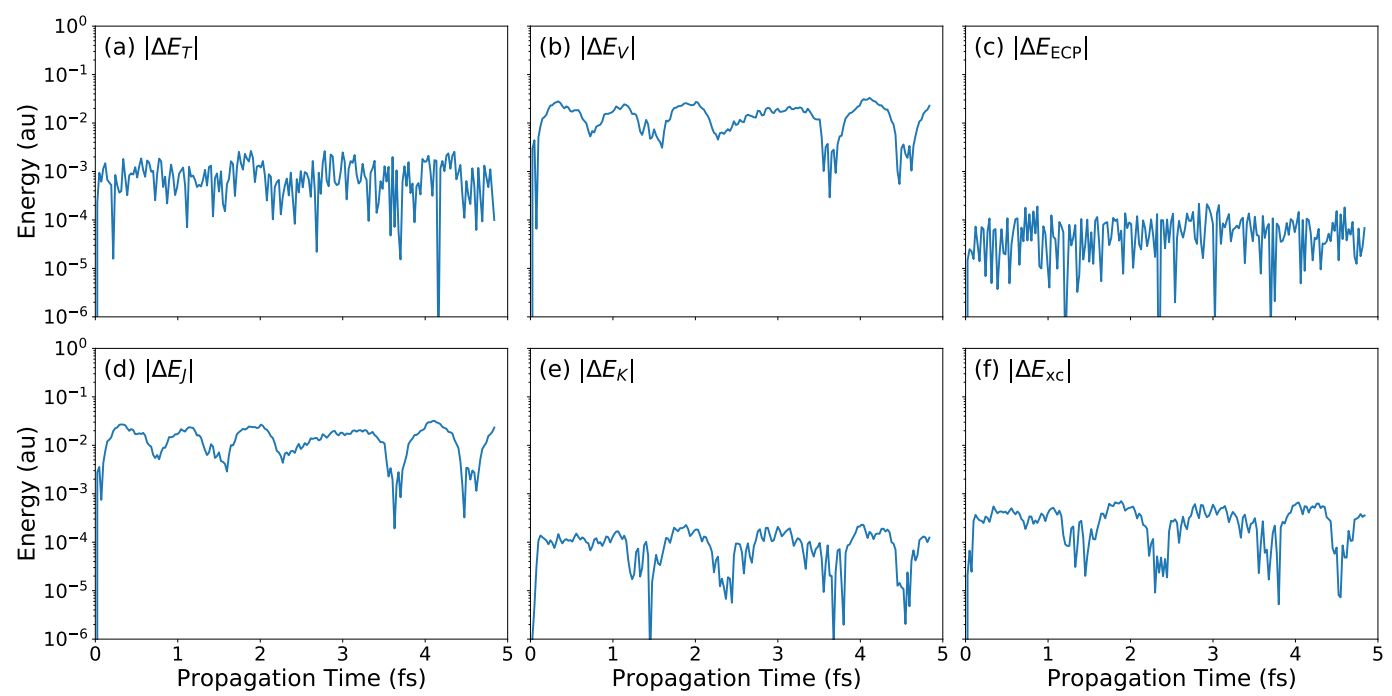

Figure S3: Fluctuation in different energy components along the RT-TDDFT trajectory for the stacked long complex. 

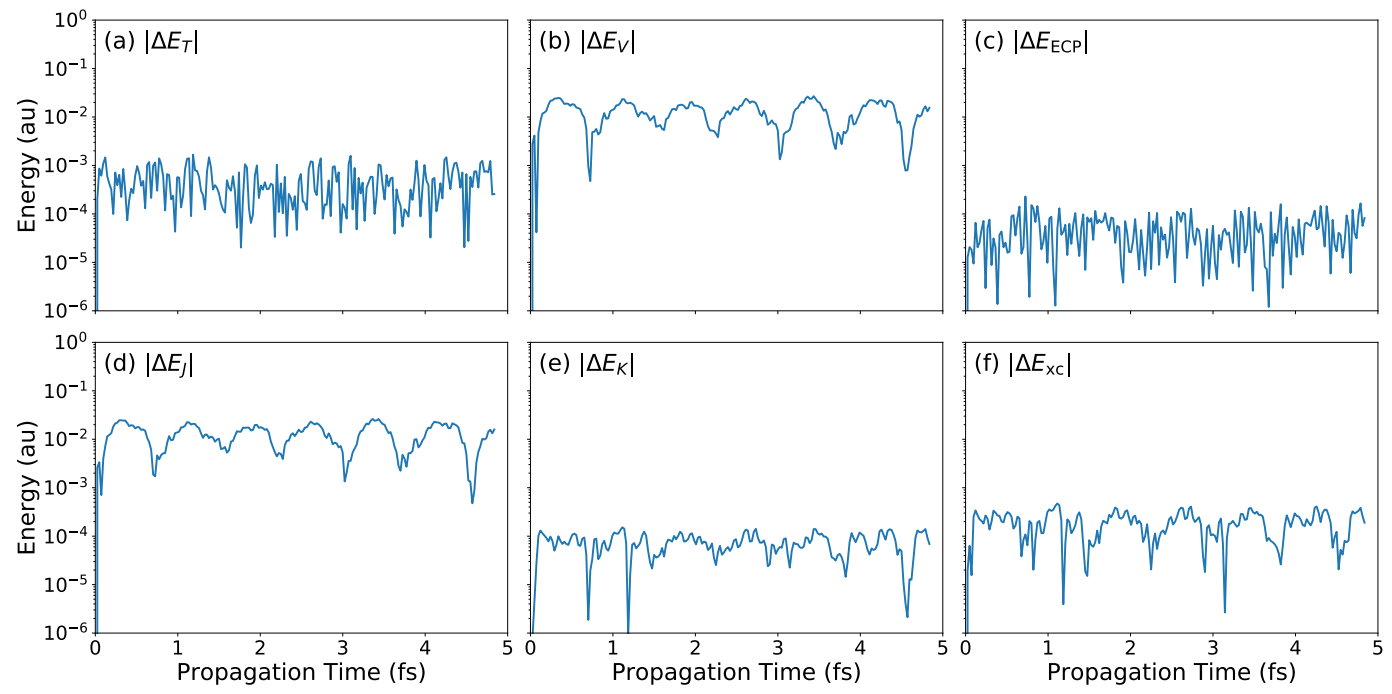

Figure S4: Fluctuation in different energy components along the RT-TDDFT trajectory for the stacked short complex.
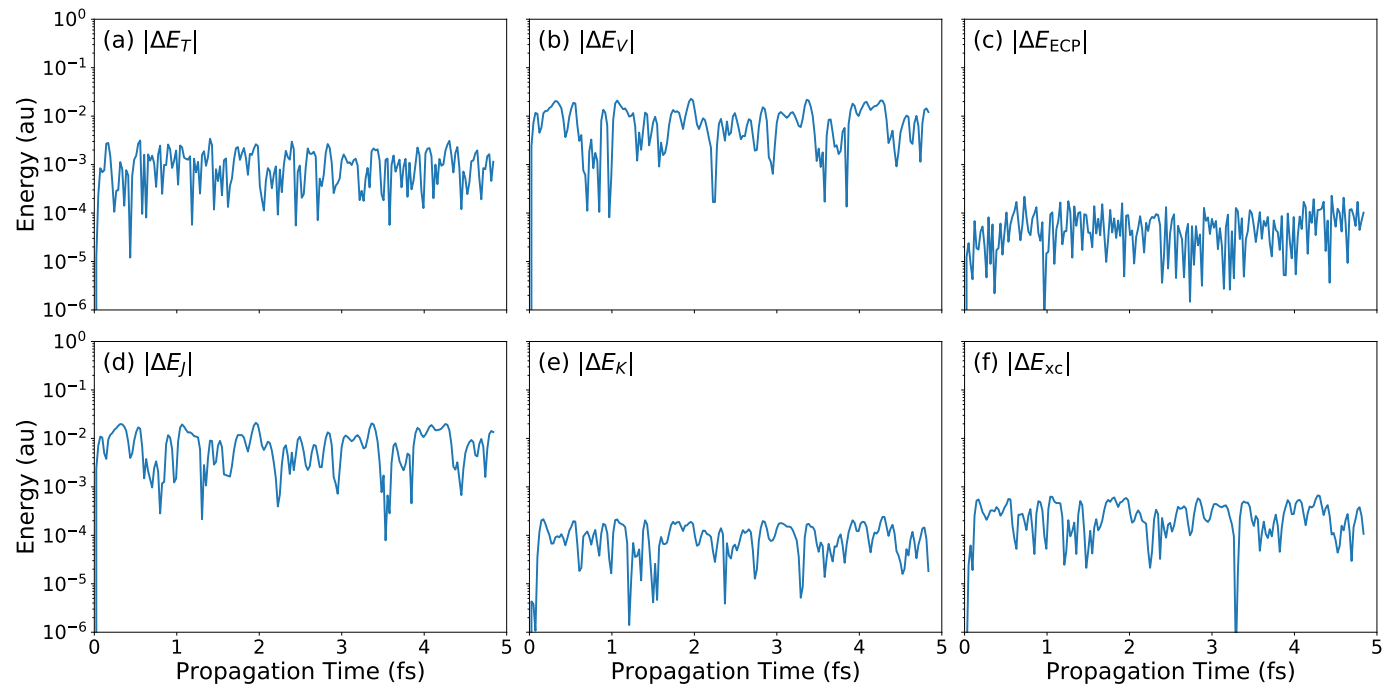

Figure S5: Fluctuation in different energy components along the RT-TDDFT trajectory for the T-shaped complex. 


\section{Initial Superposition}

Table S1: Key excited states for the stacked-long complex with their excitation energies $\omega^{(m)}$, oscillation periods $T$, transition dipole moments $\mu_{x}^{(0, m)}$. Also shown are their contributions to the initial orbital rotation $(t=0)$, in terms of the expansion coefficients, $\tilde{a}_{m}(0)$ (computed using Eq. A15), $\tilde{b}_{m}(0)$ (computed using a density matrix equivalent of Eq. A16), and relative weight $p_{m}(0)$ (defined in Eq. A19), and the ratio of $a_{m}(0)$ values computed using Eqs. A15 and A12. Only states with a relative weight, $p_{m}>0.01$, are shown. The total weight $\sum_{m} p_{m}$ is 0.87 for the first 200 excited states.

\begin{tabular}{l|ccccccc}
\hline State & $\omega^{(m)}(\mathrm{au})$ & $T(\mathrm{fs})$ & $\mu_{x}^{(0, m)}($ Debye $)$ & $\tilde{a}_{m}(0)$ & $\tilde{b}_{m}(0)$ & $p_{m}(0)$ & $\tilde{a}_{m}(0) / a_{m}(0)$ \\
\hline 5 & 0.1263 & 1.2032 & -0.8268 & -0.0134 & -0.0121 & 0.1926 & 1.0243 \\
10 & 0.1570 & 0.9678 & -0.3005 & -0.0040 & -0.0036 & 0.0168 & 1.0435 \\
15 & 0.1646 & 0.9232 & -1.1224 & -0.0135 & -0.0121 & 0.1947 & 0.9909 \\
20 & 0.1726 & 0.8808 & 0.4158 & 0.0048 & 0.0042 & 0.0242 & 0.9964 \\
34 & 0.1999 & 0.7601 & 1.6921 & 0.0169 & 0.0139 & 0.2778 & 0.9984 \\
46 & 0.2099 & 0.7242 & -0.9663 & -0.0092 & -0.0075 & 0.0824 & 0.9988 \\
93 & 0.2573 & 0.5907 & -0.4590 & -0.0036 & -0.0024 & 0.0101 & 0.9990 \\
95 & 0.2579 & 0.5893 & 0.5411 & 0.0042 & 0.0028 & 0.0141 & 1.0011 \\
\hline
\end{tabular}

Table S2: Key excited states for the stacked-short complex with their excitation energies $\omega^{(m)}$, oscillation periods $T$, transition dipole moments $\mu_{x}^{(0, m)}$. Also shown are their contributions to the initial orbital rotation $(t=0)$, in terms of the expansion coefficients, $\tilde{a}_{m}(0)$ (computed using Eq. A15), $\tilde{b}_{m}(0)$ (computed using a density matrix equivalent of Eq. A16), and relative weight $p_{m}(0)$ (defined in Eq. A19), and the ratio of $a_{m}(0)$ values computed using Eqs. A15 and A12. Only states with a relative weight, $p_{m}>0.01$, are shown. The total weight $\sum_{m} p_{m}$ is 0.86 for the first 200 excited states.

\begin{tabular}{l|ccccccc}
\hline State & $\omega^{(m)}(\mathrm{au})$ & $T(\mathrm{fs})$ & $\mu_{x}^{(0, m)}($ Debye $)$ & $\tilde{a}_{m}(0)$ & $\tilde{b}_{m}(0)$ & $p_{m}(0)$ & $\tilde{a}_{m}(0) / a_{m}(0)$ \\
\hline 12 & 0.1602 & 0.9487 & 1.3821 & 0.0173 & 0.0155 & 0.3477 & 1.0001 \\
19 & 0.1724 & 0.8816 & -0.3483 & -0.0040 & -0.0036 & 0.0188 & 1.0019 \\
34 & 0.1993 & 0.7627 & 1.7065 & 0.0171 & 0.0142 & 0.3145 & 0.9985 \\
46 & 0.2097 & 0.7247 & -0.9239 & -0.0088 & -0.0073 & 0.0831 & 0.9988 \\
90 & 0.2551 & 0.5957 & 0.6494 & 0.0051 & 0.0034 & 0.0222 & 1.0009 \\
135 & 0.2739 & 0.5548 & 0.5838 & 0.0043 & 0.0032 & 0.0179 & 0.9999 \\
178 & 0.2949 & 0.5153 & 0.6250 & 0.0043 & 0.0031 & 0.0172 & 1.0031 \\
\hline
\end{tabular}


Table S3: Key excited states for the T-shaped complex with their excitation energies $\omega^{(m)}$, oscillation periods $T$, transition dipole moments $\mu_{x}^{(0, m)}$. Also shown are their contributions to the initial orbital rotation $(t=0)$, in terms of the expansion coefficients, $\tilde{a}_{m}(0)$ (computed using Eq. A15), $\tilde{b}_{m}(0)$ (computed using a density matrix equivalent of Eq. A16), and relative weight $p_{m}(0)$ (defined in Eq. A19), and the ratio of $a_{m}(0)$ values computed using Eqs. A15 and A12. Only states with a relative weight, $p_{m}>0.01$, are shown. The total weight $\sum_{m} p_{m}$ is 0.85 for the first 200 excited states.

\begin{tabular}{l|ccccccc}
\hline State & $\omega^{(m)}(\mathrm{au})$ & $T(\mathrm{fs})$ & $\mu_{x}^{(0, m)}($ Debye $)$ & $\tilde{a}_{m}(0)$ & $\tilde{b}_{m}(0)$ & $p_{m}(0)$ & $\tilde{a}_{m}(0) / a_{m}(0)$ \\
\hline 6 & 0.1261 & 1.2049 & -0.7612 & -0.0124 & -0.0113 & 0.1597 & 1.0288 \\
10 & 0.1559 & 0.9747 & 0.2850 & 0.0038 & 0.0034 & 0.0148 & 1.0457 \\
13 & 0.1626 & 0.9346 & 1.2075 & 0.0147 & 0.0132 & 0.2214 & 0.9926 \\
20 & 0.1721 & 0.8832 & -0.3785 & -0.0044 & -0.0039 & 0.0193 & 0.9987 \\
34 & 0.1995 & 0.7618 & -1.7067 & -0.0171 & -0.0141 & 0.2740 & 0.9983 \\
46 & 0.2095 & 0.7255 & 0.9502 & 0.0091 & 0.0075 & 0.0768 & 0.9987 \\
94 & 0.2577 & 0.5897 & 0.6447 & 0.0050 & 0.0035 & 0.0196 & 1.0003 \\
\hline
\end{tabular}

\section{Geometries}

\subsection{Stacked-long}

Energy values (in Hartree) and nuclear coordinates (in $\AA$ ) of silver-nitrogen configurations using the PBE0/6-31G(d) level of theory,

\section{6}

the total energy is -697.274292 au

$\begin{array}{llll}\mathrm{Ag} & -0.00000 & -1.31103 & 0.00000\end{array}$

$\begin{array}{llll}\mathrm{Ag} & -0.00000 & 0.00000 & 2.40747\end{array}$

$\begin{array}{llll}\mathrm{Ag} & 0.00000 & -0.00000 & -2.40747\end{array}$

$\begin{array}{llll}\mathrm{Ag} & 0.00000 & 1.31103 & -0.00000\end{array}$

$\begin{array}{llll}\mathrm{N} & 3.90000 & 0.00000 & 0.55978\end{array}$

$\begin{array}{llll}\mathrm{N} & 3.90000 & 0.00000 & -0.55978\end{array}$

\subsection{Stacked-short}

Energy values (in Hartree) and nuclear coordinates (in $\AA$ ) of silver-nitrogen configurations using the PBE0/6-31G(d) level of theory,

6

the total energy

$\mathrm{Ag} \quad-0.00000$

$\mathrm{Ag} \quad-0.00000$

$\mathrm{Ag} \quad 0.00000$

$\mathrm{Ag} \quad 0.00000$

$\mathrm{N} \quad 3.90000$

$\mathrm{N} \quad 3.90000$

$\begin{array}{rr}\text { is } \quad-697.274533 & \\ -1.31103 & 0.00000 \\ 0.00000 & 2.40747 \\ -0.00000 & -2.40747 \\ 1.31103 & -0.00000 \\ 0.55978 & 0.00000 \\ -0.55978 & 0.00000\end{array}$




\section{$4.3 \quad$ T-shape}

Energy values (in Hartree) and nuclear coordinates (in $\AA$ ) of silver-nitrogen configurations using the PBE0/6-31G(d) level of theory,

6

the total energy

$\mathrm{Ag} \quad-0.00000$

$\mathrm{Ag} \quad-0.00000$

$\mathrm{Ag} \quad 0.00000$

$\mathrm{Ag} \quad 0.00000$

$\mathrm{N} \quad 4.75978$

$\mathrm{N} \quad 3.64022$

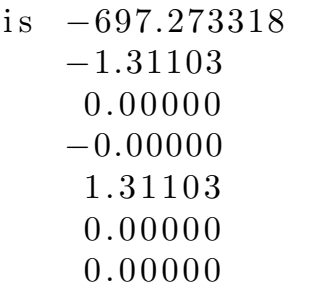

0.00000

2.40747

$-2.40747$

$-0.00000$

0.00000

0.00000 